\title{
HARMONIC MAPPINGS BETWEEN SINGULAR METRIC SPACES
}

\author{
CHANG-YU GUO \\ Dedicated to Professor Pekka Koskela on the occasion of his $60^{\text {th }}$ birthday
}

\begin{abstract}
In this paper, we survey the existence, uniqueness and interior regularity of solutions to the Dirichlet problem associated to various energy functionals in the setting of mappings between singular metric spaces. Based on known ideas and techniques, we separate the necessary analytical assumptions to axiomatizing the theory in the singular setting. More precisely,

- We extend the existence result of Guo and Wenger [30] for solutions to the Dirichlet problem of Korevaar-Schoen energy functional to more general energy functionals in purely singular setting.

- When $Y$ has non-positive curvature in the sense of Alexandrov (NPC), we show that the ideas of Jost [45] and Lin [59] can be adapted to the purely singular setting to yield local Hölder continuity of solutions of the Dirichlet problem of KorevaarSchoen and Kuwae-Shioya.

- We extend the Liouville theorem of Sturm [74] for harmonic functions to harmonic mappings between singular metric spaces.

- We extend the theorem of Mayer [64] on the existence of the harmonic mapping flow and solve the corresponding initial boundary value problem.

Combing these known ideas, with the more or less standard techniques from analysis on metric spaces based on upper gradients, leads to new results when we consider harmonic mappings from $\operatorname{RCD}(K, N)$ spaces into NPC spaces. Similar results for the Dirichlet problem associated to the Kuwae-Shioya energy functional and the upper gradient functional are also derived.
\end{abstract}

\section{CONTENTS}

1. Introduction 2

1.1. Existence and uniqueness 3

1.2. Interior regularity 6

1.3. Liouville theorem 8

1.4. Harmonic mapping flow 8

$\begin{array}{ll}\text { 2. Preliminaries } & 10\end{array}$

2.1. Metric spaces of non-positive curvature 10

$\begin{array}{ll}2.2 \text {. Strong measure contraction properties } & 10\end{array}$

Date: May 11, 2021.

2010 Mathematics Subject Classification. 58E20,46E35,53C23,31C25.

Key words and phrases. Dirichlet problem, Sobolev space, harmonic mapping, harmonic mapping flow, NPC space, ultra-completion.

C.-Y. Guo was supported by Swiss National Science Foundation Grant 175985 and the Qilu funding of Shandong University (No. 62550089963197). 
2.3. Ultra-completions of metric spaces 12

2.4. Metric space valued Sobolev spaces 12

2.5. Cheeger energy and $\operatorname{RCD}(K, N)$ spaces 15

3. Existence and uniqueness of solutions to the Dirichlet problem of Korevaar $\begin{array}{ll}\text { and Schoen } & 16\end{array}$

3.1. Formulation of Dirichlet problem associated to an energy functional $\mathcal{E} \quad 16$

3.2. Energy functionals with property $\mathcal{B} \quad 16$

3.3. Proof of Theorem 1.1 18

3.4. Proof of Theorem 1.5 20

4. Hölder Regularity of harmonic mappings 22

4.1. Dirichlet forms 22

4.2. Approximating energy densities with Property $\mathcal{C} \quad 23$

4.3. Proof of Theorem 1.6 1). 30

4.4. Proof of Theorem 1.6 2) 34

5. A Liouille type theorem for harmonic mappings 37

5.1. Composition with distance function 37

5.2. Proof of Theorem $1.8 \quad 40$

6. Harmonic mapping flow 41

Acknowledgements $\quad 42$

$\begin{array}{ll}\text { References } & 42\end{array}$

\section{INTRODUCTION}

Given a mapping $u: M \rightarrow N$ between two smooth Riemannian manifolds, there is a natural concept of energy associated to $u$. The minimizers, or more generally, the critical points of such energy functional, are called harmonic mappings. In the very beginning, the research on harmonic mappings comes together with the theory of minimal surfaces and it has attracted great attention after the work of Bochner [70]. However, the important existence, uniqueness and regularity theory were established relatively late - only after the work of Morrey [65] on the Plateau problem in Riemannian manifold. The breakthrough in higher dimensional theory of harmonic mappings was made by Eells and Sampson [19], Hartman [32] and idenpendently by Alber [1, 2] and by Hamilton [31] for manifolds with boundary via the heat equation method, where the target manifold $N$ was assumed to be non-positively curved. The regularity theory for general target Riemannian manifold has later been developed by Schoen and Uhlenbeck in a seminal paper [67] and independently in $[25,26]$ for manifolds with a single chart; see also $[68,37,47]$. In the remarkable work of Gromov and Schoen [29], the authors proposed a variational approach for the theory of harmonic mappings to the setting of mappings into singular metric spaces, along with important applications to rigidity problems for certain discrete groups.

Now, consider a mapping $u: X \rightarrow Y$, where $X=(X, d, \mu)$ is a metric measure space and $Y=(Y, d)$ a metric space. In a fundamental and important paper of Korevaar and Schoen [51], an energy functional associated to $L^{2}(X, Y)$ mappings was introduced. More 
precisely, for each $\varepsilon>0$, one defines an approximating energy functional $E_{\varepsilon}(u): C_{0}(X) \rightarrow$ $\mathbb{R}$ on the space of continuous functions with compact support by

$$
E_{\varepsilon}(u)(f)=\int_{X} f(x) f_{B(x, \varepsilon)} \frac{d(u(x), u(y))^{2}}{\varepsilon^{2}} d \mu(y) d \mu(x) .
$$

In case $X$ is a compact $C^{2}$-smooth Riemannian manifold or a relatively compact domain in a $C^{2}$-smooth Riemannian manifold, it was shown that $E_{\varepsilon}(u)$ converges weakly, as a positive linear functional on $C_{0}(X)$, to some energy functional $E(u)$. Based on this energy functional, which we refer as Korevaar-Schoen energy functional, they have successfully extended the theory of harmonic mappings from $C^{2}$-smooth Riemannian manifolds into metric spaces with non-positive curvature in the sense of Alexandrov (NPC). Independently, Jost introduced in [42] a slightly different energy functional and developed a theory of harmonic mappings associated to that energy functional through a sequential of deep works [42, 43, 44, 45, 46]. Moreover, the existence result of Jost [42] works for mappings defined on more general metric spaces than domains in $C^{2}$-smooth Riemannian manifolds. From now on, if not specified, harmonic mappings refer to the energy minimizers of the Korevaar-Schoen energy functional.

Since then, there has been a considerable amount of growing interest in the theory of harmonic mappings between singular spaces. In particular, in the research monograph of Eells-Fuglede [18], the authors extended the theory of harmonic mappings to the setting where $X$ is an admissible Riemannian polyhedron. Gregori [28] further extended the existence and uniqueness theory of harmonic mappings to the setting where $X$ is a Lipschitz Riemannian manifold. Capogna and Lin [12] extended part of the harmonic mapping theory to the setting of mappings from Euclidean spaces to the Heisenberg groups. In a series of deep works [76, 77, 78], Sturm developed a theory of harmonic mappings (associated to a slightly different functional) via a probabilistic theory and the theory of (generalized) Dirichlet forms.

1.1. Existence and uniqueness. There are two general approaches for the existence of harmonic mappings: the first one relies on the uniform convexity of the distance function in NPC spaces (see e.g. [51, 28, 18, 22]), while the second one relies on the theory of (metric space valued) Sobolev mappings, in particular, the theory of trace, lower semicontinuity of the enery functional with respect to the $L^{2}$-convergence and the (various versions of) Rellich compactness theorem (see e.g. [62, 30]). In the first approach, a crucial fact one needed is the so-called subpartition lemma, which essentially says that the integral averages one uses to approximate the Sobolev energy satisfy certain monotonicity with respect to the size of the ball on which the average is taken. The advantage of this approach is that we can solve the Dirichlet problem of Korevaar and Schoen for fairly general open subset of the metric measure space $X$; see for instance [22]. In the second approach, the domain $\Omega \subset X$ has to be sufficiently nice so that both the theory of trace and certain version of Rellich compactness theorem holds. The advantage of the second approach is that the target metric space $Y$ does not need to NPC, and indeed, can be fairly 
general, which includes in particular all proper metric spaces, all dual Banach spaces and NPC spaces; see [30]. Of course, one cannot expect uniqueness in such a great generality.

Our first main result of this paper concerns the existence of harmonic mappings into singular metric spaces. Note that both our source domain and the target metric space are fairly general.

Theorem 1.1. Let $(X, d, \mu)$ be a compact metric space and $Y$ a metric space that is 1 complemented in some ultra-completion of $Y$. Suppose $X$ admits an energy functional $\mathcal{E}$ with property $\mathcal{B}$. Fix a domain $\Omega \subset X$. Then, for each $\phi \in S^{1,2}(X, Y)$, there exists a mapping $u \in S_{\phi}^{1,2}(\Omega, Y)$ such that

$$
\mathcal{E}(u)=\inf _{v \in S_{\phi}^{1,2}(\Omega, Y)} \mathcal{E}(v) .
$$

As observed in [30, Proposition 2.1], many nice metric spaces are 1-complemented in some ultra-completion of themselves, in particular, proper metric spaces, dual Banach spaces, $L^{1}$-spaces and NPC spaces. The definition of metric spaces that admits an energy functional $\mathcal{E}$ with property $\mathcal{B}$ shall be given in Section 3.2 below. It is stated in a very abstract sense.

As the Korevaar-Schoen energy functional $E$ has property $\mathcal{B}$, we obtain, as a direct corollary of Theorem 1.1, the existence of Dirichlet problem of Korevaar-Schoen.

Corollary 1.2. Suppose $(X, d, \mu)$ is compact $\operatorname{RCD}(K, N)$ and $Y$ is a metric space that is 1-complemented in some ultra-completion of $Y$. Then for each $\phi \in K S^{1,2}(X, Y)$, there exists a mapping $u \in K S_{\phi}^{1,2}(\Omega, Y)$ such that

$$
E(u)=\inf _{v \in K S_{\phi}^{1,2}(\Omega, Y)} E(v) .
$$

Kuwae and Shioya [57] constructed an energy functional which slightly differs from the Korevaar-Schoen energy functional. They introduced a new concept, called strong measure contraction property of Bishop-Gromov type (SMCPBG) (given in Section 2.2 below), and developed a theory of Sobolev space of mappings $u: X \rightarrow Y$ when the source metric measure space space $X$ satisfies the SMCPBG. Riemannian manifolds and Alexandrov spaces with curvature bounded from below are typical examples of metric measure spaces that posse the SMCPBG. Since the Kuwae-Shioya energy functional $E^{b}$ has property $\mathcal{B}$, we have the following corollary.

Corollary 1.3. Suppose $(X, d, \mu)$ is a compact metric space satisfying the $S M C P B G$ and $Y$ is a metric space that is 1-complemented in some ultra-completion of $Y$. Then for each $\phi \in W^{1,2}(X, Y)$, there exists a mapping $u \in W_{\phi}^{1,2}(\Omega, Y)$ such that

$$
E^{b}(u)=\inf _{v \in W_{\phi}^{1,2}(\Omega, Y)} E^{b}(v)
$$

Parallel to the theory of harmonic mappings, great effort has been made to extend the existence, uniqueness and regularity theory of harmonic functions, that is harmonic mappings into the real line $Y=\mathbb{R}$, to the abstract metric measure space setting; see for instance $[14,72,50,54,33,41,9]$ and the references therein. Unlike the case of 
mappings, one usually uses the $L^{2}$-norm of the upper gradients as the energy functional. Since the notion of upper gradients works also for mappings, it is natural to consider the Dirichlet problem associated to the energy functional of upper gradients. As a by-product of Theorem 1.1, we obtain the following existence result for the Dirichlet problem based on upper gradients.

Corollary 1.4. Suppose $(X, d, \mu)$ is a compact PI space and $Y$ is a metric space that is 1-complemented in some ultra-completion of $Y$. Then for each $\phi \in N^{1,2}(X, Y)$, there exists a mapping $u \in N_{\phi}^{1,2}(\Omega, Y)$ such that

$$
E^{g}(u)=\inf _{v \in N_{\phi}^{1,2}(\Omega, Y)} E^{g}(v) .
$$

Recall that we say a metric measure space $X=(X, d, \mu)$ a PI-space, if the measure $\mu$ is doubling on $X$, i.e., there exists a constant $c_{d}>0$ such that

$$
\mu(B(x, 2 r)) \leq c_{d} \mu(B(x, r))
$$

for all open balls $B(x, r) \subset X$ with $\operatorname{diam} B \leq \operatorname{diam} X$ and it supports a weak $(1,2)$ Poincaré inequality (2.8). Corollary 1.4 can be regarded as a natural extension of [72, Theorem 5.6] to the setting of metric space valued mappings. Note however that the proof of Theorem 5.6 in [72] cannot work in this case since $N^{1,2}(X, Y)$ is not a linear (Banach) space for general metric target.

The idea for the proof of Theorem 1.1 relies on that used in [30, Proof of Theorem 1.4] but some more care needs to be paid in order to deal with the singular/irregular source domain. The main difference with the situation there is that we do not have a theory of trace for metric space valued Sobolev mapping as that of [51]. Instead, we (essentially) use the fact that admissible mappings are defined on a (relatively compact) neighborhood of the domain $\Omega$ and coincide with a given boundary value. In the first step, we apply the generalized compactness result (see [30, Theorem 3.1]) to obtain an energy minimizing harmonic mapping into some ultra-completion $Y_{\omega}$ of the target metric space $Y$. Composing this map with the 1-Lipschitz projection map from $Y_{\omega}$ to $Y$, we then obtain an energy minimizing harmonic mapping from $X$ to $Y$. The only remaining issue is to show that this map has the correct boundary value, which will follow by our construction of the map into the ultra-completion.

Regarding the uniqueness, we have the following result for the Korevaar-Schoen energy functional.

Theorem 1.5. Suppose $(X, d, \mu)$ is $\operatorname{RCD}(K, N)$ and $Y$ is $N P C$. Fix a relatively compact domain $\Omega \subset X$ that supports a $(1,2)$-Poincaré inequality (3.2) and that has the property $\operatorname{Cap}_{2}(X \backslash \Omega)>0$. Then, for each $\phi \in K S^{1,2}(X, Y)$, there exists a unique mapping $u \in$ $K S_{\phi}^{1,2}(\Omega, Y)$ such that

$$
E(u)=\inf _{v \in K S_{\phi}^{1,2}(\Omega, Y)} E(v) .
$$

The general idea of the proof of Theorem 1.5 is quite similar to [51, Proof of Theorem 2.2], but relies on the theory of metric-valued Sobolev spaces developed in [35]. In 
particular, we provide a new and concrete proof of [22, Theorem 1 (a)] in a greater generality. Moreover, if $X$ is instead assumed to satisfy the SMCPBG, then the conclusion of Theorem 1.5 holds with the Kuwae-Shioya energy functional $E^{b}$; see Remark 3.9.

1.2. Interior regularity. In their fundamental work [51], Korevaar and Schoen has shown that harmonic mappings from a $C^{2}$-smooth Riemannian manifold to an NPC space is locally Lipschitz continuous, which plays an important role in establishing rigidity theorems of geometric group theory (see e.g. $[29,16]$ ). Since then, there has been a lot of effort in establishing interior regularity of harmonic mappings in the singular space setting; see for instance [15, 73, 43, 45, 21, 22, 23, 40, 16, 82, 38, 39, 83, 62] and the references therein. It should be noticed that one cannot expect local Lipschitz continuity holds in general singular metric spaces. Indeed, Chen [15] constructed a harmonic function on a two-dimensional metric cone $X$ such that $u$ is not Lipschitz continuous if $X$ has no lower curvature bound. Nevertheless, harmonic functions constructed there do satisfy the local Hölder continuity, which is valid for all harmonic mappings from admissible Riemannian polyhedrons to NPC spaces (see e.g. [15, 18]).

In [59], Lin proposed a very elegant approach to obtain Hölder continuity of harmonic mappings between singular spaces. In particular, Lin's method implies that harmonic mappings from Alexandrov spaces with curvature bounded from below to locally doubling NPC spaces are local Hölder continuous, provided that the composition of the distance function with the harmonic mapping is subharmonic. As observed in [82], the latter requirement holds for harmonic mappings from Alexandrov spaces with curvature bounded from below to NPC spaces via an argument due to Jost [45]. Note that in the work of Jost [45], a fairly general interior Hölder regularity result for harmonic mappings (associated to the Jost's energy functional) was established. On the other hand, as pointed out in [55], the approach of Jost [45] relies on a theory of generalized Dirichlet forms for metric space valued mappings, which is in general hard to verify since the classical method of Beurling and Deny (see e.g. [24]) fails in constructing energy measures for metric-space valued mappings. However, the essential ingredients in Jost's regularity result are volume doubling property and (2,2)-Poincaré inequality for the intrinsic metric space (induced by the Dirichlet form) and hence it can be extended to rather general setting.

Our second main purpose of this paper is to establish interior Hölder regularity of Korevaar-Schoen and Kuwae-Shioya harmonic mappings in a large class of singular metric spaces. Our second main result reads as follows.

Theorem 1.6. 1). Let $X$ be a complete metric measure space and $Y$ a locally doubling $N P C$ space. If the approximating energy density $e_{\varepsilon}^{c, Y}$ between $X$ and $Y$ has property $\mathcal{C}$, then each solution $u$ of the Dirichlet problem (if exists) is locally Hölder continuous.

2). Let $X$ be a complete metric measure space and $Y$ an NPC space. If the approximating energy density $e_{\varepsilon}^{c, Y}$ between $X$ and $Y$ has strong property $\mathcal{C}$, then each solution $u$ of the Dirichlet problem (if exists) is locally Hölder continuous.

The definition of approximating energy density with (strong) property $\mathcal{C}$ shall be given in Section 4.2 below. For a given NPC space $Y$, when $X$ is either the Lipschitz manifold 
considered in [28], or the admissible Riemannian polyhedron considered in [15, 18, 17], or metric spaces with the strong measure contraction property (SMCP) considered in [75] or $\operatorname{RCD}(K, N)$-space considered in [5, 6, 27], the Korevaar-Schoen approximating energy density between $X$ and $Y$ will have strong property $\mathcal{C}$. Similarly, when $X$ satisfies the SMCPBG, the Kuwae-Shioya approximating energy density will have strong property $\mathcal{C}$; see Examples 4.5 and 4.6 below. Consequently, we obtain the following corollary of Theorem 1.6.

Corollary 1.7. Let $Y$ be an NPC space. Then we have

- when $X$ is $\operatorname{RCD}(K, N)$, each solution $u$ of the Dirichlet problem of KorevaarSchoen (if exists) is locally Hölder continuous;

- when X satisfies the SMCPBG, each solution u of the Dirichlet problem of KuwaeShioya (if exists) is locally Hölder continuous.

The proof of Theorem 1.6 1) is a combination of the approach of Jost [45] and Lin [59]. More precisely, we first follow the idea of Jost to show that for each point $y_{0} \in Y$, the function $f_{y_{0}}:=d^{2}\left(u(\cdot), y_{0}\right.$ ) is (weakly) subharmonic in the sense of [8] (in terms of Dirichlet forms), and then adapt the argument of Lin [59] to prove the local Hölder regularity. We would like to point out that the argument of Lin [59] is elegant and beautiful, but it requires the target space to be (locally) doubling. Thus it cannot be applied in general NPC targets.

The proof of Theorem 1.6 2) follows closely the approach of Jost [45]. The essential difference with [45] is that we work directly on the Dirichlet forms for functions, instead of the generalized Dirichlet forms for mappings as in [45], whereas the well-known regularity theory of sub/super solutions associated to Dirichlet forms developed in [8] can be applied.

We did not address the interior regularity for the case when $Y$ is a CAT(1) space in this paper. It is worth pointing out that in this more general case similar interior regularity results have been obtained when $X$ is an admissible Riemannian polyhedron in [21, 23] or when $X$ is an Alexandrov space with curvature bounded from below in [39, 81].

Note that Theorem 1.6 implies that harmonic mappings (associated to the KorevaarSchoen energy functional) from $\operatorname{RCD}(K, N)$ space to NPC space are locally Hölder continuous. In a recent remarkable work of Zhang and Zhu [82] (see also [83]), harmonic mappings were shown to be locally Lipschitz continuous, when the source metric measure space is an Alexandrov space with curvature bounded from below and the target metric space is NPC. Since Alexandrov spaces with curvature bounded from below are special cases of $\operatorname{RCD}(K, N)$-spaces, this and the corresponding result for harmonic functions [41] seem to suggest that Korevaar-Schoen harmonic mappings may be locally Lipschitz continuous, if the metric measure space $X$ is $\operatorname{RCD}(K, N)$; see [83, Problem 4.6] for more detailed discussions on this interesting open problem.

Open problem. Can we establish the interior Höler continuity of solutions for the Dirichlet problem associated to the upper gradient energy functional?

The main difficulty to this open problem is that it is very difficult to compute the upper gradient of a composed function and thus we cannot use the approach for Theorem 1.6 to 
infer the composition of the distance function with $u$ will be any kind of "subharmonic" function.

1.3. Liouville theorem. Liouville type theorems for harmonic mappings between complete smooth Riemannian manifolds have been investigated by many authors including geometers and probabilists. In particular, Eells-Sampson [19] proved that any bounded harmonic mapping from a compact Riemannian manifold with positive Ricci curvature into a complete manifold with non-positive curvature is constant. Schoen and Yau [69] proved that any harmonic mapping with finite energy from a complete smooth Riemannian manifolds with non-negative Ricci curvature into a complete manifold with non-positive curvature is constant. Cheng [13] showed any harmonic mapping with sublinear growth from a complete Riemannian manifold with non-negative Ricci curvature into an Hadamard manifold is constant. Hildebrandt-Jost-Widman [36] proved a Liouville type theorem for harmonic mappings into regular geodesic balls in a complete smooth Riemannian manifold. For a detailed description of other types of Liouville type theorems for harmonic mappings; see [58].

For the statement of our Liouville theorem for harmonic mappings, we set for $u: X \rightarrow$ $Y$,

$$
v_{p}\left(r, x, x_{0}\right)=\int_{B(x, r)} d\left(u(x), u\left(x_{0}\right)\right)^{p} d \mu(x)
$$

Theorem 1.8. Let $X$ be a complete metric measure space and $Y$ an NPC space. Suppose the approximating energy density $e_{\varepsilon}^{c, Y}$ between $X$ and $Y$ has property $\mathcal{C}$. If $u \in$ $K S_{\text {loc }}^{1,2}(X, Y)$ is a harmonic mapping such that for some $x, x_{0} \in X$ and $p>1$,

$$
\int_{1}^{\infty} \frac{r}{v_{p}\left(r, x, x_{0}\right)} d r=\infty
$$

then $u$ is constant.

The proof of Theorem 1.8 relies on the Liouville type theorem for weakly subharmonic functions. Originally, Yau [80, Theorem 1] has shown that there is no non-constant smooth non-negative $L^{p}$-integrable, $p>1$, subharmonic functions on a complete smooth Riemannian manifold. Sturm [74, Theorem 1] extended this result to the setting of Dirichlet forms under the sharper condition (1.1). In our setting, Theorem 1.8 is a direct application of this Liouville theorem for weakly subharmonic functions. More precisely, we shall show that $v=d\left(u(\cdot), u\left(x_{0}\right)\right)$ is a weakly subharmonic function (in the sense of Sturm [74]) on $X$ and so by [74, Theorem 1], $v$ is constant. Consequently, $u=u\left(x_{0}\right)$ is constant. In the proof of $v$ being weakly subharmonic, we shall combine the idea of Jost [45] together with the theory of fine topology and potential theory that developed recently in the setting of metric spaces $[9,10]$.

1.4. Harmonic mapping flow. In [64], Mayer developed a general theory of gradient flows on NPC spaces with successful applications to harmonic mapping. The basic setting is an NPC space $L=(L, D)$ together with an energy functional $G: L \rightarrow \mathbb{R} \cup\{\infty\}$. The 
gradient flow equation

$$
\frac{d u(t)}{d t}=-\nabla G(u(t))
$$

has been interpreted as a variational formulation:

$$
u(t+h) \quad \text { minimizes } \quad u \mapsto G(u)+\frac{1}{2 h} D^{2}(u, u(t)) .
$$

The existence and uniqueness of $u(t+h)$ were obtained under mild structural assumptions on $G$; see [28, Theorem 1.13] and [48].

In our setting, it is not difficult to show that $L=L^{2}(X, Y)$ is NPC when $X$ is complete and $Y$ is NPC (see Lemma 6.1). On the other hand, when $X$ has strong property $\mathcal{B}$, $u \mapsto E(u)$ is a lower semicontinuous convex functional. Thus as an immediate corollary of [64, Theorem 1.13], we obtain the following result.

Theorem 1.9 (Existence and boundedness of gradient flow of the Dirichlet Energy). Assume $X$ is $\operatorname{RCD}(K, N)$ and $Y$ is $N P C$. For any starting point $u_{0} \in K S^{1,2}(X, Y)$ the gradient flow of the Korevaar-Schoen energy exists and $u_{t} \in K S^{1,2}(X, Y)$ for each $t \geq 0$. Moreover, if $X$ has finite $\mu$-measure, then the gradient flow stays bounded for all times.

As an immediate corollary of Theorem 1.9 and the Rellich compactness theorem for Sobolev mappings, we obtain the following corollary.

Corollary 1.10. In the setting of Theorem 1.9, if $X$ is compact and $Y$ is proper, then the flow $u_{t}$ converges to a constant mapping as $t \rightarrow \infty$.

As an easy consequence of our proof of Theorem 1.1, we can show that the Sobolev space $\mathcal{K S}_{\phi}^{1,2}(\Omega, Y)$ is NPC and consequently solve the initial boundary value problem via the exactly same method as in [64, Theorem 3.4].

Theorem 1.11 (Solvability of the initial boundary value problem). Assume $X$ is $\operatorname{RCD}(K, N)$ and $Y$ is NPC. For any given map $\phi \in K S^{1,2}(\Omega, Y)$, the following problem admits a solution in the sense of [64, Theorem 1.13]:

$$
\left\{\begin{array}{l}
u(t) \text { solves the harmonic mapping flow for } t \geq 0, \\
u(0)=\phi, \\
\text { a representative of } u(t) \text { equal } \phi \text { q.e. in } X \backslash \Omega .
\end{array}\right.
$$

Moreover, if $\Omega$ is relatively compact in $X$, then $\hat{u}=\lim _{t \rightarrow \infty} u(t)$ exists and is the unique harmonic mapping solving the Dirichlet problem of Korevaar and Schoen with boundary data $\phi$.

Sturcture of the paper. This paper is structured as follows. In section 2, we recall the necessary definitions needed for this paper, mainly, the definition of Sobolev spaces of metric-valued mappings. In section 3, we prove the existence and uniqueness theorem. In section 4, we show the interior Hölder continuity of harmonic mappings. In section 5, we prove the Liouville theorem for harmonic mappings. We study the associated harmonic mapping flow in Section 6. In the final section, Section ??, we study the Dirichlet problem 
associated to other energy functionals, in particular, the energy functional of Kuwae and Shioya and the energy functional based on upper gradients.

\section{Preliminaries}

The metric space $Y$ in this paper is always assumed to be separable so that we may embeds $Y$ isometrically into the Banach space $l^{\infty}(Y)$.

2.1. Metric spaces of non-positive curvature. We shall need the following concept, which was introduced by Alexandrov [3]; see also [7].

Definition 2.1 (NPC spaces). A complete metric space $(X, d)$ (possibly infinite dimensional) is said to be non-positively curved (NPC) if the following two conditions are satified:

- $(X, d)$ is a length space, that is, for any two points $P, Q$ in $X$, the distance $d(P, Q)$ is realized as the length of a rectifiable curve connecting $P$ to $Q$. (We call such distance-realizing curves geodesics.)

- For any three points $P, Q, R$ in $X$ and choices of geodesics $\gamma_{P Q}$ (of length $r$ ), $\gamma_{Q R}$ (of length $p$ ), and $\gamma_{R P}$ (of length q) connecting the respective points, the following comparison property is to hold: For any $0<\lambda<1$, write $Q_{\lambda}$ for the point on $\gamma_{Q R}$ which is a fraction $\lambda$ of the distance from $Q$ to $R$. That is,

$$
d\left(Q_{\lambda}, Q\right)=\lambda p, \quad d\left(Q_{\lambda}, R\right)=(1-\lambda) p .
$$

On the (possibly degenerate) Euclidean triangle of side lengths $p, q, r$ and opposite vertices $\bar{P}, \bar{Q}, \bar{R}$, there is a corresponding point

$$
\bar{Q}_{\lambda}=\bar{Q}+\lambda(\bar{R}-\bar{Q}) .
$$

The NPC hypothesis is that the metric distance $d\left(P, Q_{\lambda}\right)$ (from $Q_{\lambda}$ to the opposite vertex $P)$ is bounded above by the Euclidean distance $\left|\bar{P}-\bar{Q}_{\lambda}\right|$. This inequality can be written precisely as

$$
d^{2}\left(P, Q_{\lambda}\right) \leq(1-\lambda) d^{2}(P, Q)+\lambda d^{2}(P, R)-\lambda(1-\lambda) d^{2}(Q, R) .
$$

In an NPC space $Y$, geodesics connecting each pair of points are unique and so one can define the $t$-fraction mapping $u_{t}$ of two mapping $u_{0}, u_{1}: X \rightarrow Y$ as $u_{t}=$ " $(1-t) u_{0}+t u_{1}$ ", that is, for each $x, u_{t}(x)$ is the unique point $P$ on the geodesic connecting $u_{0}(x)$ and $u_{1}(x)$ such that $d\left(P, u_{0}(x)\right)=t d\left(u_{0}(x), u_{1}(x)\right)$ and $d\left(P, u_{1}(x)\right)=(1-t) d\left(u_{0}(x), u_{1}(x)\right)$. We refer the interested readers to [51, Section 2.1] or [46] for more discussions on NPC spaces.

2.2. Strong measure contraction properties. The following notion of measure contraction property was introduced by Sturm [75].

Definition 2.2 (Weak measure contraction property). We say that a metric measure space $(X, d, \mu)$ satisfies the weak measure contraction property (WMCP) if there exist numbers $R>0, \theta<\infty$ and $\lambda<\infty$ and $\mu^{2}$-measurable maps $\Phi_{t}: X \times X \rightarrow X$ with the following properties: 
(1) For $\mu$-a.e. $x_{1}, x_{2} \in X$ with $d\left(x_{1}, x_{2}\right)<R$ and all $s, t \in[0,1]$,

$$
\Phi_{0}(x, y)=x, \Phi_{t}(x, y)=\Phi_{1-t}(y, x), \Phi_{s}\left(x, \Phi_{t}(x, y)\right)=\Phi_{s t}(x, y)
$$

and

$$
d\left(\Phi_{s}(x, y), \Phi_{t}(x, y)\right) \leq \lambda|s-t| d(x, y)
$$

(2) For all $\varepsilon<R$, $\mu$-a.e. $x \in X$, all $\mu$-measurable set $A \subset B(x, r)$ and all $t \in[0,1]$,

$$
\frac{\mu_{\varepsilon}(A)}{\sqrt{\mu(B(x, r))}} \leq \theta \frac{\mu_{t \varepsilon}\left(\Phi_{t}(x, A)\right)}{\sqrt{\mu(B(x, t \varepsilon))}},
$$

where $d \mu_{\varepsilon}(x)=\frac{d \mu(x)}{\sqrt{\mu(B(x, \varepsilon))}}$ and $d \mu_{\varepsilon}(y)=\frac{d \mu(y)}{\sqrt{\mu(B(y, \varepsilon))}}$.

Definition 2.3 (SMCP). We say that a metric measure space $(X, d, \mu)$ posses the strong measure contraction property (SMCP) if it satisfies the WMCP and the constants $\Theta$ and $\theta$ appearing in Definition 2.2 can be chosen to be arbitrarily close to 1.

Many interesting metric spaces satisfies SMCP. In particular, $C^{2}$-smooth Riemannian $n$-manifolds; see [75, Section 4] for more examples.

Definition 2.4 (WMCPBG). We say that a metric measure space $(X, d, \mu)$ satisfies the weak measure contraction property of Bishop-Gromov type (WMCPBG) if there exist $\nu>$ 0 , and increasing continuous function $b:[0, \infty) \rightarrow[0, \infty)$ with $b(0)=0$ such that there are positive finite constants $R, \Theta \geq 1$ and $\theta \geq 1$, and $\mu \otimes \mu$-measurable mappings $\Phi_{t}=$ $\Phi_{t}^{Z}: X \times X \rightarrow X$ for all $t \in[0,1]$, with the following

i). For $\mu$-a.e. $x, y \in X$ with $d(x, y)<R$ and all $s, t \in[0,1]$,

$$
\begin{gathered}
\Phi_{0}(x, y)=x, \Phi_{t}(x, y)=\Phi_{1-t}(y, x), \Phi_{s}\left(x, \Phi_{t}(x, y)\right)=\Phi_{s t}(x, y) \\
d\left(\Phi_{s}(x, y), \Phi_{t}(x, y)\right) \leq \theta|t-s| d(x, y) .
\end{gathered}
$$

ii). For all positive $r<R$, $\mu$-a.e. $x \in X$, all $\mu$-measurable set $A \subset B(x, r)$ and all $t \in[0,1]$,

$$
\frac{\mu(A)}{b(r)} \leq \Theta \frac{\mu\left(\Phi_{t}(x, A)\right)}{b(r t)} .
$$

iii). For all positive $r<R, \mu$-a.e. $x \in X$,

$$
\mu(B(x, r)) \leq \theta b(r) .
$$

iv). For $0<r_{1} \leq r_{2} \leq R \theta^{2}$,

$$
\frac{b\left(r_{2}\right)}{b\left(r_{1}\right)} \leq \Theta\left(\frac{r_{2}}{r_{1}}\right)^{\nu}
$$

Definition 2.5 (SMCPBG). We say that a metric measure space $(X, d, \mu)$ posses the strong measure contraction property of Bishop-Gromov type (SMCPBG) if it satisfies the $W M C P B G$ and the constants $\Theta$ and $\theta$ appearing in Definition 2.4 can be chosen to be arbitrarily close to 1.

Example 2.6. The following spaces satisfy the $S M C P B G$ property. 
1). $C^{2}$-smooth Riemannian manifolds $\left(M, d_{g}, \mu_{g}\right)$ satisfies $S M C P B G$ with $b(r)=$ $\omega_{n-1} r^{n} / n$.

2). Let $X$ be an $n$-dimensional Alexandrov space with curvature bounded from below by some $\kappa \in \mathbb{R}$. Then $X$ has the $S M C P B G$ with $\mu=\mathcal{H}^{n}$ and $b(r)=\omega_{n-1} r^{n} / n$.

More interesting examples can be found in [75, Section 4].

2.3. Ultra-completions of metric spaces. We briefly recall the relevant definitions concerning ultra-completions and ultra-limits of metric spaces. Details can be found for instance in [11].

A non-principal ultrafilter on $\mathbb{N}$ is a finitely additive probability measure $\omega$ on $\mathbb{N}$ such that every subset of $\mathbb{N}$ is measurable and such that $\omega(A)$ equals 0 or 1 for all $A \subset \mathbb{N}$ and $\omega(A)=0$ whenever $A$ is finite. Given a compact Hausdorff topological space $(Z, \tau)$ and a sequence $\left\{z_{m}\right\} \subset Z$ there exists a unique point $z_{\infty} \in Z$ such that $\omega\left(\left\{m \in \mathbb{N}: z_{m} \in U\right\}\right)=1$ for every $U \ni \tau$ containing $z_{\infty}$. We denote the point $z_{\infty}$ by $\lim _{\omega} z_{m}$.

Let $Y=(Y, d)$ be a metric space and $\omega$ a non-principal ultrafilter on $\mathbb{N}$. A sequence $\left\{y_{m}\right\} \subset Y$ is bounded if $\sup _{m} d\left(y_{1}, y_{m}\right)<\infty$. Define an equivalence relation $\sim$ on bounded sequences in $Y$ by considering $\left\{y_{m}\right\}$ and $\left\{y_{m}^{\prime}\right\}$ equivalent if $\lim _{\omega} d\left(y_{m}, y_{m}^{\prime}\right)=0$. Denote by $\left[\left(y_{m}\right)\right]$ the equivalence class of $\left\{y_{m}\right\}$. The ultra-completion $Y_{\omega}$ of $Y$ with respect to $\omega$ is the metric space given by the set

$$
Y_{\omega}:=\left\{\left[\left(y_{m}\right)\right]:\left\{y_{m}\right\} \text { bounded sequence in } Y\right\},
$$

equipped with the metric

$$
d_{\omega}\left(\left[\left(y_{m}\right)\right],\left[\left(y_{m}^{\prime}\right)\right]\right):=\lim _{\omega} d\left(y_{m}, y_{m}^{\prime}\right) .
$$

The ultra-completion $Y_{\omega}$ of $Y$ is a complete metric space, even if $Y$ itself is not complete.

Following [30], a metric space $Y$ is said to be 1-complemented in some metric space $Z$ if $Y$ isometrically embeds into $Z$ and if there exists a 1-Lipschitz retraction from $Z$ to $Y$. By [30, Proposition 2.1], the class of metric spaces $Y$ which are 1-complemented in every ultra-completion of $Y$ includes proper metric spaces, NPC spaces, dual Banach spaces and injective metric spaces.

\subsection{Metric space valued Sobolev spaces.}

2.4.1. Korevaar-Schoen Sobolev spaces. For each $\varepsilon>0$, we define an approximating energy $E_{\varepsilon}(u): C_{0}(X) \rightarrow \mathbb{R}$ by

$$
E_{\varepsilon}(u)(f)=\int_{X} f_{B(x, \varepsilon)} f(x) \frac{d(u(x), u(y))^{2}}{\varepsilon^{2}} d \mu(x) d \mu(y),
$$

Definition 2.7. (Korevaar-Schoen-Sobolev space) Let $u \in L^{2}(X, Y)$. We say $u$ is in the Korevaar-Schoen-Sobolev space $K S^{1,2}(X, Y)$ if

$$
E(u):=\sup _{\varphi \in C_{c}(X,[0,1])} \limsup _{\varepsilon \rightarrow 0} E_{\varepsilon}(u)(\varphi)
$$

is finite. For $u \in K S^{1,2}(X, Y), E(u)$ is the Korevaar-Schoen energy functional of $u$. 
2.4.2. Kuwae-Shioya Sobolev spaces. As in the definition of Korevaar-Schoen Sobolev spaces, we first introduce the approximating energy.

Definition 2.8 (Approximating energy). Fix a positive function $f \in C_{0}(X)$ and an admissible rate function $b$. For each $u \in L^{p}(X, Y)$, we set

$$
E_{\varepsilon}^{b}(u)(f):=\frac{1}{2 b(\varepsilon)} \int_{X} f(x) \int_{B(x, \varepsilon)} \frac{d(u(x), u(y))^{2}}{\varepsilon^{2}} d \mu(y) d \mu(x) .
$$

If $X$ satisfies the SMCPBG, then it follows from [57, Theorem 3.1] that for each $f \in C_{0}(X)$, the limit

$$
E^{b}(u)(f):=\lim _{\varepsilon \rightarrow 0} E_{\varepsilon}^{b}(u)(f)
$$

exists. The limit functional $E^{b}(u)$ is called the Kuwae-Shioya energy functional of $u$.

Definition 2.9 (Kuwae-Shioya energy). The Kuwae-Shioya energy of $u$ is defined to be

$$
E^{b}(u):=\sup _{0 \leq f \leq 1, f \in C_{0}(X)} E^{b}(u)(f) .
$$

The Sobolev space of Kuwae-Shioya is then defined to be

$$
W^{1,2}(X, Y)=\left\{u \in L^{2}(X, Y): E^{b}(u)<\infty\right\} .
$$

2.4.3. Sobolev spaces based on upper gradients. Let $X=(X, d, \mu)$ be a metric measure space. Let $\Gamma$ a family of curves in $X$. A Borel function $\rho: X \rightarrow[0, \infty]$ is admissible for $\Gamma$ if for every locally rectifiable curves $\gamma \in \Gamma$,

$$
\int_{\gamma} \rho d s \geq 1
$$

The 2-modulus of $\Gamma$ is defined as

$$
\operatorname{Mod}_{2}(\Gamma)=\inf _{\rho}\left\{\int_{X} \rho^{2} d \mu: \rho \text { is admissible for } \Gamma\right\} .
$$

A family of curves is called 2-exceptional if it has 2-modulus zero. We say that a property of curves holds for 2-almost every curve if the collection of curves for which the property fails to hold is 2-exceptional.

Let $X=(X, d, \mu)$ be a metric measure space and $Z=\left(Z, d_{Z}\right)$ be a metric space. A Borel function $g: X \rightarrow[0, \infty]$ is called an upper gradient for a map $u: X \rightarrow Z$ if for every rectifiable curve $\gamma:[a, b] \rightarrow X$, we have the inequality

$$
\int_{\gamma} g d s \geq d_{Z}(u(\gamma(b)), u(\gamma(a)))
$$

If inequality (2.6) holds for 2-almost every curve, then $g$ is called a 2-weak upper gradient for $u$.

The concept of upper gradient was first introduced in [34] and then functions with 2-integrable upper gradients were studied in [53]. Later, the theory of real-valued Sobolev spaces based on upper gradients was explored in-depth in [71]. 
A 2-weak upper gradient $g$ of $u$ is minimal if for every 2-weak upper gradient $\tilde{g}$ of $u$, $\tilde{g} \geq g \mu$-almost everywhere. If $u$ has an upper gradient in $L_{\mathrm{loc}}^{2}(X)$, then $u$ has a unique (up to sets of $\mu$-measure zero) minimal 2-weak upper gradient. We denote the minimal upper gradient by $g_{u}$. The Newtonnian Sobolev space $N^{1,2}(X)$ consists of all functions $u \in L^{2}(X)$ with a minimal 2-weak upper gradient $g_{u} \in L^{2}(X)$. The notation $N_{0}^{1,2}(X)$ represents the class of all functions $g \in N^{1,2}(X)$ with compact support.

Definition 2.10 (Sobolev capacity). The 2-capacity of a set $E \subset X$ is defined to be the (possibly infinite) number

$$
\operatorname{Cap}_{2}(E)=\inf \left(\int_{X}|u|^{2}+g_{u}^{2} d \mu\right)
$$

where the infimum is taken over all functions $u \in N^{1, p}(X)$ such that $u \geq 1$ outside a 2-exceptional set of measure zero.

Each function $u \in N^{1,2}(X)$ is quasicontinuous, meaning that for every $\varepsilon>0$, there exists an open set $G_{\varepsilon} \subset X$ with $\operatorname{Cap}_{2}\left(G_{\varepsilon}\right)<\varepsilon$ such that the restriction $\left.u\right|_{X \backslash G_{\varepsilon}}$ is continuous. Let $u, v: X \rightarrow Y$ be two mappings. We say that $u=v$ quasi-everywhere or q.e. in $X$ if $\operatorname{Cap}_{2}(\{x \in X: u(x) \neq v(x)\})=0$.

We say that a metric measure space $X=(X, d, \mu)$ supports a weak (1,2)-Poincaré inequality if there exist constants $C \geq 1$ and $\tau \geq 1$ such that

$$
f_{B}\left|u-u_{B}\right| d \mu \leq C \operatorname{diam}(B)\left(f_{\tau B} g^{2} d \mu\right)^{1 / 2}
$$

for all open balls $B$ in $X$, for every function $u: X \rightarrow \mathbb{R}$ that is integrable on balls and for every upper gradient $g$ of $u$ in $X$.

The Sobolev capacity enjoys many nice properties, such as the Cartan, Choquet and Kellogg properties, making it very useful in the theory of fine topology in Euclidean spaces; see for instance [63, Section 2.4]. These nice properties of Sobolev 2-capactiy remain valid in complete metric spaces that are doubling and support a weak $(1,2)$-Poincaré inequality; see e.g. [10, Section 7] and [9, Chapter 11].

As an immdeiate consequence of the Choquet property of 2-capacity, one obtains that finely open sets are quasiopen. Here, a set $U \subset X$ is quasiopen if for every $\varepsilon>0$, there is an open set $G \subset X$ such that $\operatorname{Cap}_{2}(G)<\varepsilon$ and $G \cup U$ is open; see [10] for the definition of finely open sets and fine topology, and proofs of the above fact in the metric space setting. The aforementioned fact implies the following lemma, which substitutes for the partition of unity in metric spaces. This lemma was first proved (in a slightly stronger form) in [49, Lemma 2.4] in Euclidean spaces and the proof remain valid in metric spaces with the help of the above fact; see also [63, Lemma 2.153].

Lemma 2.11. Let $X$ be a complete doubling metric space that supports a weak (1,2)Poincaré inequality. Let $\left\{U_{\alpha}\right\}_{\alpha}$ be a covering of an open set $U \subset X$ with each $U_{\alpha}$ being quasiopen. For each positive $g \in N_{0}^{1,2}(U)$, there exists a sequence of positive functions $g_{j}$ converging to $g$ in $N_{0}^{1,2}(U)$ such that each $g_{j}$ is a finite sum of functions in $N_{0}^{1,2}\left(U_{\alpha}\right)$. 
By [9, Theorem 11.40], every quasicontinuous function $g: U \rightarrow[-\infty, \infty]$ is finely continuous at q.e. $x \in U$, which means that $g$ is continuous when $U$ equipped with the fine topology and $\bar{R}$ with the usual toplogy. In particular, the level sets of each function $g \in N^{1,2}(X)$ are finely open and thus quasiopen if $X$ is complete doubling with a weak $(1,2)$-Poincaré inequality.

The definition of metric space-valued Newtonnian Sobolev spaces $N^{1,2}(X, Y)$ can be found in [35, Chapter 7.1] and we do not recall it here. For each $u \in N^{1,2}(X, Y)$, we shall use $E^{g}(u)$ to denote the upper gradient energy functional of $u$, that is,

$$
E^{g}(u)=\int_{X} g_{u}^{2} d \mu
$$

2.4.4. Equivalence of different Sobolev spaces. There are also other definitions of metric space valued Sobolev spaces. Under mild assumptions for the source space $X$, one can show that all these definitions of Sobolev spaces coincide; see [35, Chapter 10]. For our purpose, we shall point out a few facts on the relation of Korevaar-Schoen and KuwaeShioya Sobolev spaces with Newtonnian Sobolev spaces.

The following result shows the connection between $K S^{1,2}(X, Y)$ and $N^{1,2}(X, Y)$; see [35, Theorem 10.4.3 and Corollary 10.4.6].

Theorem 2.12. Assume that $X$ is doubling. Then each $u \in K S^{1,2}(X, Y)$ has a $\mu$ representative $\tilde{u}$ in $N^{1,2}(X, Y)$ satisfying

$$
E^{g}(\tilde{u}) \leq C E(u)
$$

where the constant $C$ depends only on the doubling constant of $\mu$. If in addition $X$ supports a weak (1,2)-Poincaré inequality, then each $u \in N^{1,2}(X, Y)$ belongs to $K S^{1,2}(X, Y)$.

The following lemma is a simple consequence of [57, Theorem 4.2] and [35, Theorem 10.3.4].

Lemma 2.13. If $X$ is a compact metric space satisfying the $S M C P B G$, then there exists a constant $C$, depending only on the doubling constant of $X$ and the constant associated to the weak Poincaré inequality, such that for each $u \in W^{1,2}(X, Y)$, we have

$$
E^{b}(u) \leq C E^{g}(u) .
$$

In particular, $W^{1,2}(X, Y) \subset N^{1,2}(X, Y)$ (up to $\mu$-representative).

Proof. Since $X$ is compact, it follows from [57, Theorem 4.2] that $X$ is doubling with constant $c_{X}$ and supports a weak $(2,2)$-Poincaré inequality with constant $C_{X}$. In particular, each $u \in W^{1,2}(X)$ belongs to $P^{1,2}(X)$, the Sobolev space defined via weak $(1,2)$ Poincaré inequality (see e.g. [35, Section 10.3]). The claim follows then from [35, Theorem 10.3.4].

2.5. Cheeger energy and $\operatorname{RCD}(K, N)$ spaces. In the celebrated works [60, 79], the notion of a lower bound on the Ricci curvature was introduced for general metric measure spaces. These spaces were termed $\operatorname{CD}(K, N)$ or $\mathrm{CD}(K, \infty)$ spaces, where the word $\mathrm{CD}$ refers to curvature-dimension, as it was defined via optimal transportation. In another 
significant work [4], a rich theory of (Sobolev) calculus was developed on such spaces. In particular, for each $u \in N^{1,2}(X)$, the Cheeger energy is defined as

$$
\operatorname{Ch}(u):=\inf \left\{\liminf _{n \rightarrow \infty} \frac{1}{2} \int_{X}\left|D u_{n}\right|^{2} d \mu: u_{n} \in \operatorname{Lip}_{b}(X), u_{n} \rightarrow u \text { in } L^{2}(X)\right\} .
$$

One weakness for such spaces is that the Cheeger energy functional is not necessarily a quadratic form. In [5], the authors introduced the stronger notion of a lower bound on the Riemannian Ricci curvature on a general metric measure space. Such metric spaces were called $\operatorname{RCD}(K, N)$ or $\operatorname{RCD}(K, \infty)$ spaces. Roughly speaking, $\operatorname{RCD}(K, N)$ spaces are $\mathrm{CD}(K, N)$ plus the requirement that the Cheeger energy function $\mathbf{C h}$ is quadratic; see $[5,6]$ for more information about these spaces.

\section{Existence And uniqueness of solutions to the Dirichlet PRoblem of KOREVAAR AND SCHOEN}

\subsection{Formulation of Dirichlet problem associated to an energy functional $\mathcal{E}$.} Fix a mapping $\phi$ in a Sobolev space $S^{1,2}(X, Y)$ with energy functional $\mathcal{E}$ and we set

$$
S_{\phi}^{1,2}(\Omega, Y)=\left\{u \in S^{1,2}(X, Y) \text { : a } \mu \text {-reprentative of } u \text { equals } \phi \text { q.e. in } X \backslash \Omega\right\} \text {. }
$$

In practice, we shall choose $\left(S^{1,2}(X, Y), \mathcal{E}\right)$ to be the Korevaar-Schoen Sobolev space $\left(K S^{1,2}(X, Y), E\right)$, the Kuwae-Shioya Sobolev space $\left(W^{1,2}(X, Y), E^{b}\right)$, or the Newtonnian Sobolev space $\left(N^{1,2}(X, Y), E^{g}\right)$. As was pointed out in Section 2, these Sobolev spaces coincide and the corresponding energy functionals are comparable on compact subsets.

Recall that the space $N_{0}^{1,2}(\Omega)$ consists of functions $u \in N^{1,2}(X)$ such that $u=0$ quasi-everywhere in $X \backslash \Omega$. We may equivalently characterize mappings in $S_{\phi}^{1,2}(\Omega, Y)$ as those mappings $u \in S^{1,2}(X, Y)$ that has a $\mu$-representative $\hat{u}$ for which $d(\hat{u}, \phi) \in N_{0}^{1,2}(\Omega)$. Indeed, if $u \in S_{\phi}^{1,2}(\Omega, Y)$, then a $\mu$-representative $\hat{u}$ of $u$ satisfies $d(\hat{u}, \phi) \in N^{1,2}(X)$ and $d(\hat{u}, \phi)=0$ quasi-everywhere in $X \backslash \Omega$, which implies that $d(\hat{u}, \phi) \in N_{0}^{1,2}(\Omega)$. On the other hand, if $u \in S^{1,2}(X, Y)$ has a $\mu$-representative $\hat{u}$ such that $d(\hat{u}, \phi) \in N_{0}^{1,2}(\Omega)$, then $\hat{u}=\phi$ quasi-everywhere in $X \backslash \Omega$ and so $u \in S_{\phi}^{1,2}(\Omega, Y)$.

We say that a domain $\Omega \subset X$ supports a $(1,2)$-Poincaré inequality if there exits a constant $C_{\Omega}>0$ such that

$$
\int_{\Omega}|v(x)| d \mu(x) \leq C_{\Omega}\left(\int_{\Omega}\left|g_{v}(x)\right|^{2} d \mu(x)\right)^{1 / 2}
$$

for all functions $v \in N_{0}^{1,2}(\Omega)$.

\subsection{Energy functionals with property $\mathcal{B}$.}

Definition 3.1 (Property $\mathcal{B})$. We say that an energy functional $\mathcal{E}$ between a complete metric measure space $X=(X, d, \mu)$ and a metric space $Y$ has property $\mathcal{B}$ if the following conditions are satisfied:

(B1): The Sobolev space $S^{1,2}(X, Y)$ is equivalent with $N^{1,2}(X, Y)$ and there exists a constant $C \geq 1$ such that for each $u \in S^{1,2}(X, Y)$

$$
C^{-1} \mathcal{E}(u) \leq E^{g}(u) \leq C \mathcal{E}(u) .
$$


(B2): The metric measure space $(X, d, \mu)$ is locally a PI-space: for each relatively compact domain $K \subset X$, there exist a constant $c_{K} \geq 1$ and a radius $R_{K}>0$

$$
\mu(B(x, 2 r)) \leq c_{d} \mu(B(x, r))
$$

for each open ball $B(x, r) \subset K$ with $r<R_{K} / 2$. Moreover, $X$ supports a the following local $(1,2)$-Poincaré inequality, for each compact set $K \subset X$, there exist $C_{K}>0$ and $\lambda_{K} \geq 1$ such that,

$$
f_{B}\left|u(x)-u_{B}\right| d \mu(x) \leq C_{K} \operatorname{diam} B\left(f_{\lambda_{K} B} g^{2} d \mu(x)\right)^{1 / 2},
$$

for all open balls $B$ in $K$ with $\lambda_{k} B \subset K$, for every function $u: X \rightarrow \mathbb{R}$ that is integrable on balls and for every upper gradient $g$ of $u$ in $X$.

(B3): The energy functional $\mathcal{E}$ is lower semicontinuous with respect to the $L^{2}$-convergence, that is, if $u_{i} \rightarrow u$ in $L^{2}(X, Y)$, where $u_{i}$ and $u$ belong to $S^{1,2}(X, Y)$, then

$$
\mathcal{E}(u) \leq \liminf _{i \rightarrow \infty} \mathcal{E}\left(u_{i}\right)
$$

We would like to point out that condition (B2) above, volume doubling and weak $(1,2)$-Poincaré inequality, were initially introduced in [45] as one of the basic conditions to build up a Hölder regularity theory for harmonic mappings in the singular space setting.

For the Korevaar-Schoen energy functional $E$ and the Kuwae-Shioya energy functional $E^{b}$, we introduce a slightly stronger notion.

Definition 3.2 (Strong property $\mathcal{B})$. We say that the Korevaar-Schoen energy functional $E$ between a complete metric space $X$ and a metric space $Y$ has strong property $\mathcal{B}$ if $E$ has property $\mathcal{B}$ and if in addition for each $u \in K S^{1,2}(X, Y)$ and each $f \in C_{0}(X)$, the pointwise limit $E(u)(f)=\lim _{\varepsilon \rightarrow 0} E_{\varepsilon}(u)(f)$ exists. The definition for the Kuwae-Shioya energy functional $E^{b}$ is similar by replacing $E$ with $E^{b}$.

For Lipschitz manifolds considered in [28], admissible Riemannian polyhedrons considered in $[15,18,17]$ and metric spaces with the SMCP considered in [75], the KorevaarSchoen energy functional $E$ has strong property $\mathcal{B}$.

Example 3.3. On the following metric measure spaces $X$, the Korevaar-Schoen energy functional $E$ between $X$ and any metric space $Y$ have strong property $\mathcal{B}$.

1). $X$ is an admissible Riemannian polyhedron.

2). $X$ satisfies the $S M C P$.

3). $X$ is an $n$-dimensional Lipschitz submanifold of $\mathbb{R}^{N}$.

4). $X$ is $\operatorname{RCD}(K, N)$ in the sense of $[27]^{1}$.

Proof. 1). On admissible Riemannian polyhedrons, $E$ has strong property $\mathcal{B}$ follows from [18, Section 9].

\footnotetext{
${ }^{1}$ By the same reasoning, one can show that the strongly rectifiable metric spaces introduced in [27] has property $\mathcal{B}$. Since we do not know any example other than $\operatorname{RCD}(K, N)$ spaces that is strongly rectifiable, we shall not consider these spaces in this article.
} 
2). By [75, Proposition 4.5 and Theorem 6.4], $(X, d, \mu)$ is locally doubling and supports a local $(2,2)$-Poincaré inequality, and hence it satisfies (B1) and (B2). The lower semicontinuity, property (B3), is a consequence of [57, Proof of Theorem 3.2]. The additionally required property follows from the proofs of Theorem 3.1 and Theorem 4.1 in [57] by using the sub-partition lemma [75, Lemma 5.2].

3). Property (B1) and (B2) are immediate as being a PI-space and Property (B3) follows from [28, Theorem 2]. The additionally required property is a direct consequence of $[28$, Theorem 1].

4). Property (B1) and (B2) follow from [66, 79] and Property (B3) follows from [27, Theorem 4.16]. The additionally required property follows from [27, Theorem 3.13].

Regarding the other energy functionals, we have the following results.

Example 3.4. 1). If $(X, d, \mu)$ is a compact metric space satisfying the $S M C P B G$, then the Kuwae-Shioya energy functional $E^{b}$ between $X$ and any metric space $Y$ has strong property $\mathcal{B}$.

2). If $(X, d, \mu)$ is a compact PI space, then the upper gradient energy functional $E^{g}$ betwen $X$ and any metric space $Y$ has property $\mathcal{B}$.

Proof. 1). Note that property (B1) and (B2) follow from [57, Theorem 4.2] and the lower semicontinuity, property (B3), is a consequence of [57, Theorem 3.2]. The additionally required property is a direct consequence of [57, Theorem 3.1 and Theorem 4.1].

2). Properties (B1) and (B2) are clear. The lower semicontinuity follows from [35, Theorem 7.3.9].

Remark 3.5. We would like to point out that it is very difficult, in general, to determine whether an energy functional $\mathcal{E}$ between a given metric measure space $X$ and a metric space $Y$ has property $\mathcal{B}$ or not and often it requires a very nice geometric structure on $X$ (but not necessarily for $Y$ ) in order to admit such a nice energy functional.

3.3. Proof of Theorem 1.1. We will need the following compactness result from [30, Theorem 3.1] in our existence proof below.

Theorem 3.6 (Generalized Rellich compactness). Suppose $\mathcal{E}$ is an energy functional between a compact metric measure space $X$ and a metric space $Y$ that has property $\mathcal{B}$. Let $\left\{u_{m}\right\} \subset K S^{1,2}(X, Y)$ be a sequence such that

$$
\sup _{k \in \mathbb{N}} \int_{X} d^{2}\left(u_{k}(x), y_{0}\right) d \mu(x)+\mathcal{E}\left(u_{k}\right)<\infty
$$

for some $y_{0} \in Y$. Then after possibly passing to a subsequence, there exist a complete metric space $Z$, isometric embeddings $\varphi_{k}: Y \rightarrow Z$, and $v \in S^{1,2}(X, Z)$ such that $\varphi_{k} \circ u_{k}$ converges to $v$ in $L^{2}(X, Z)$.

Remark 3.7. Note that in [30, Theorem 3.1], $X$ is assumed to be a bounded Lipschitz domain in $\mathbb{R}^{n}$ and $\mathcal{E}=E$ is the Korevaar-Schoen energy functional. However, this assumption was only used to ensure that each $u \in K S^{1,2}(X, Y)$ has a $\mu$-representative $\hat{u}$ 
that satisfies the pointwise inequality

$$
d\left(\hat{u}(x), \hat{u}\left(x^{\prime}\right)\right) \leq d\left(x, x^{\prime}\right)\left(h(x)+h\left(x^{\prime}\right)\right)
$$

for some $h \in L^{2}(X)$. In our setting, this fact is well-known by property (B1) and (B2) (see e.g. [35, Theorem 10.5.2 and Theorem 10.5.3]).

Proof of Theorem 1.1. The proof follows closely the approach of [30, Proof of Theorem 1.4]. Let $\left\{u_{k}\right\} \subset S_{\phi}^{1,2}(\Omega, Y)$ be an energy minimizing sequence. Up to a $\mu$-representative, we may assume that each $h_{k}(x)=d\left(u_{k}(x), \phi(x)\right) \in N_{0}^{1,2}(\Omega)$. Since $\sup _{k} \mathcal{E}\left(h_{k}\right)<\infty$, it follows easily from property (B1) and the (1,2)-Poincaré inequality (2.8) that $\sup _{k}\left\|h_{k}\right\|_{L^{2}(X)}<$ $\infty$. Hence

$$
\sup _{k} \int_{X} d^{2}\left(u_{k}(x), y_{0}\right) d \mu(x)+\mathcal{E}\left(u_{k}\right)<\infty
$$

We may apply Theorem 3.6 to find, after possibly passing to a subsequence, a complete metric space $Z=\left(Z, d_{Z}\right)$, isometric embeddings $\varphi_{k}: Y \rightarrow Z$ and $v \in S^{1,2}(X, Z)=$ $K S^{1,2}(X, Z)$ such that $v_{k}:=\varphi_{k} \circ u_{k}$ converges in $L^{2}(X, Z)$ to $v$ as $k \rightarrow \infty$. After passing to a further subsequence, we may assume that $v_{m}$ converges almost everywhere on $X$. Let $N \subset X$ be a set of $\mu$-measure zero such that $v_{k}(z) \rightarrow v(z)$ for all $z \in X \backslash N$.

By our assumption on $Y$, there exists an ultra-completion $Y_{\omega}$ on $Y$ such that $Y$ admits a 1-Lipschitz retraction $P: Y_{\omega} \rightarrow Y$. Define a subset of $Z$ by $B:=\{v(z): z \in X \backslash N\}$. The map $\psi: B \rightarrow Y_{\omega}$, given by $\psi(v(z))=\left[\left(u_{k}(z)\right)\right]$ when $z \in X \backslash N$ is well-defined and isometric by [30, Lemma 2.2]. Since $Y_{\omega}$ is complete, there exists a unique extension of $\psi$ to $\bar{B}$, which we denote again by $\psi$. After possibly redefining the map $v$ on $N$, we may assume that $v$ has image in $\bar{B}$ and hence $v$ is an element of $K S^{1,2}(X, \bar{B})$. Now, we define a mapping by $u:=P \circ \psi \circ v$ and then $u$ belongs to $K S^{1,2}(X, Y)=S^{1,2}(X, Y)$ and satisfies

$$
E(u) \leq E(v) \leq \lim _{k \rightarrow \infty} E\left(v_{m}\right)=\lim _{k \rightarrow \infty} E\left(u_{m}\right)
$$

by the lower semicontinuity of the energy from property (B2).

It remains to show that $u=\phi$ quasi-everywhere in $X \backslash \Omega$. For each $m \in \mathbb{N}$, we write $B_{m}=\left\{v_{m}(z): z \in X \backslash N\right\}$ and define $\psi_{m}: B_{m} \rightarrow Y_{\omega}$ by $\psi_{m}\left(v_{m}(z)\right)=\left[\left(u_{m}(z)\right)\right]$ (the constant sequence). Then $\psi_{m}$ is well-defined and isometric by [30, Lemma 2.2]. Moreover, $u_{m}=P \circ \psi_{m} \circ v_{m}$. Note that for q.e. $x \in X \backslash \Omega, u_{m}(x)=\phi(x)$ for all $m \in \mathbb{N}$. So for all such point $x$ we have

$$
\begin{aligned}
d(\phi(x), u(x)) & =d\left(u_{m}(x), u(x)\right)=d\left(P \circ \psi_{m} \circ v_{m}(x), P \circ \psi \circ v(x)\right) \\
& \leq d\left(\psi_{m} \circ v_{m}(x), \psi \circ v(x)\right)=\lim _{k \rightarrow \omega} d\left(u_{m}(x), u_{k}(x)\right) \\
& =d(\phi(x), \phi(x))=0,
\end{aligned}
$$

which implies that $u(x)=\phi(x)$ q.e. in $X \backslash \Omega$. Thus $u \in S_{\phi}^{1,2}(X, Y)=K S_{\phi}^{1,2}(X, Y)$ as required. 
3.4. Proof of Theorem 1.5. The following lemma will be the key for the proof of Theorem 1.5.

Lemma 3.8. Let $X$ be a complete locally PI-space and $Y$ a metric space. Let $\left\{u_{k}\right\}$ be a sequence in $K S_{\phi}^{1,2}(X, Y)$ with uniformly bounded energy. Suppose $u_{k}$ converges in $L^{2}(X, Y)$ to some $u \in K S^{1,2}(X, Y)$. Then $u \in K S_{\phi}^{1,2}(X, Y)$.

Proof. We only need to show that a $\mu$-representative of $u$ equals $\phi$ quasi-everywhere in $X \backslash \Omega$, or equivalently, for each compact set $K \subset X, u=\phi$ q.e. in $K$. Thus we may assume without loss of generality that $X$ is a complete PI space. By embedding $Y$ isometrically into $l^{\infty}(Y)$ if necessary, we may further assume that $Y$ is (isometrically) contained in a Banach space.

Note that Theorem 2.12 implies that $g_{u_{k}}$ is bounded in $L^{2}(X)$. Taking a further subsequence if necessary, we may assume that $g_{u_{k}} \rightarrow g$ weakly in $L^{2}(X)$. By Mazur's lemma (see e.g. [35, Section 2.3]), a convex combination $\hat{g}_{i}=\sum_{k=i}^{N_{i}} a_{k i} g_{u_{k}}$ of $g_{u_{k}}$ converges to $g$ strongly in $L^{2}(X)$. Consequently, the sequence $\hat{u}_{i}=\sum_{k=i}^{N_{i}} a_{k i} u_{k}$ converges to $u$ in $L^{2}(X, Y)$ with $\hat{g}_{i}$ being a 2-weak upper gradient of $\hat{u}_{i}$. Then [35, Proposition 7.3.7] implies that $u$ has a $\mu$-representative in $N^{1,2}(X, Y)$ with each Borel representative of $g$ as a 2-weak upper gradient. Moreover, a subsequence of $\hat{u}_{i}$ converges pointwise to this representative of $u$ outside a set of 2-capacity zero. Note that in $X \backslash \Omega, u_{i}=\phi$ quasi-everywhere and so is $\hat{u}_{i}$. This implies that this $\mu$-representative of $u$ coincides with $\phi$ quasi-everywhere in $X \backslash \Omega$. In particular, $u \in K S_{\phi}^{1,2}(\Omega, Y)$.

Proof of Theorem 1.5. We will follow the approach of [51] to show the existence and uniqueness of energy minimizers.

Uniqueness: For two mappings $u, v \in K S^{1,2}(X, Y)$, we denote by $w$ the middle point mapping of $u$ and $v$. More precisely, for each $x \in X$, we set $w(x)$ to be the middle point of the geodesic connection $u(x)$ and $v(x)$. Note that $d(u, v) \in K S^{1,2}(X)$. We next show that $w \in K S^{1,2}(X, Y)$.

For $x, y \in X$, by [51, Equation (2.2iii)],

$$
\begin{aligned}
2 d^{2}(w(x), w(y)) \leq d^{2}(u(x), u(y)) & +d^{2}(v(x), v(y)) \\
- & \frac{1}{2}[d(u(y), v(y))-d(u(x), v(x))]^{2} .
\end{aligned}
$$

Integrating and averaging (3.6) on the ball $B(x, \varepsilon)$ with respect to $y$; and then multiplying by $f(x)$, where $f \geq 0$ and $f \in C_{0}(\Omega)$, then integrating with respect to $x$ and sending $\varepsilon$ to zero, we deduce from Property (B3) that

$$
2 E(w)(f) \leq E(u)(f)+E(v)(f)-\frac{1}{2} E(d(u, v))(f)
$$

or equivalently,

$$
2 E(w) \leq E(u)+E(v)-\frac{1}{2} E(d(u, v))
$$

from which we infer $w \in K S^{1,2}(X, Y)$. 
Suppose $u$ and $v$ are two solutions of the Dirichlet problem. Then the middle point mapping $w$ of $u$ and $v$ belongs to $K S_{\phi}^{1,2}(\Omega, Y)$ since $w=\phi$ quasi-everywhere in $X \backslash \Omega$. It follows that $E(w) \geq E(u)=E(v)$. By (3.7), we infer that $E(h)=0$, where $h=d(u, v)$, and so for each relatively compact domain $K$ of $X$ that contains $\Omega$, we have by property (B1) and Theorem 2.12 that

$$
\int_{K} g_{h}^{2} d \mu \leq C E(h)=0 .
$$

Since $h=0$ quasi-everywhere on $X \backslash \Omega, h=0$ quasi-everywhere on $X \backslash K$ as well and so $g_{h}=0 \mu$-a.e. on $X \backslash K$. We thus conclude that

$$
\int_{X} g_{h}^{2} d \mu \leq C E(h)=0 .
$$

In particular, $g_{h}=0 \mu$-a.e. in $X$. Since $X$ is locally a PI-space, we conclude that $h=c$ for some constant $c \in \mathbb{R} \mu$-a.e. in $X$. Since $h=0$ quasi-everywhere in $X \backslash \Omega$, we conclude that $c=0$. Consequently, $u=v \mu$-a.e. in $X$. This shows the uniqueness of solutions for the Dirichlet problem.

Existence: Let $\left\{u_{i}\right\}$ be an energy minimizing sequence for the Dirichlet problem, i.e.,

$$
\lim _{i \rightarrow \infty} E\left(u_{i}\right)=\inf _{v \in K S_{\phi}^{1,2}(\Omega, Y)} E(v)=: E_{0} .
$$

Then $u_{i j}:=d\left(u_{i}, u_{j}\right) \in N_{0}^{1,2}(\Omega)$ and the middle point mapping $w_{i j}$ of $u_{i}$ and $u_{j}$ belongs to $K S_{\phi}^{1,2}(\Omega, Y)$. Thus

$$
E\left(u_{i}\right)+E\left(u_{j}\right)-2 E\left(w_{i j}\right) \leq E\left(u_{i}\right)+E\left(u_{j}\right)-2 E_{0} .
$$

By (3.7), this implies that

$$
\int_{X} g_{u_{i j}}^{2} d \mu=\int_{\Omega} g_{u_{i j}}^{2} d \mu \leq C E\left(u_{i j}\right) \rightarrow 0 \text { as } i, j \rightarrow \infty .
$$

Note that $g_{u_{i j}}=0$ in $X \backslash \Omega$, since $u_{i j}=0$ quasi-everywhere in $X \backslash \Omega$. Since $\Omega$ supports the weak (1,2)-Poincaré inequality (3.2), we infer that $u_{i j} \rightarrow 0$ in $L^{2}(\Omega)$ and so also in $L^{2}(X, Y)$ as $i, j \rightarrow \infty$, and hence $\left\{u_{i}\right\}$ has a limit $u$ in the complete metric space $L^{2}(X, Y)$. By the lower semicontinuity of the energy functional,

$$
E(u) \leq \liminf _{i \rightarrow \infty} E\left(u_{i}\right)=E_{0}
$$

and so $u \in K S^{1,2}(X, Y)$. By Lemma 3.8, $u \in K S_{\phi}^{1,2}(X, Y)$ and so $u$ is the required energy minimizer.

Remark 3.9. 1). The proof of Theorem 1.5 above implies that the requirement that $\Omega$ supports a $(1,2)$-Poincaré inequality can be dropped if in additional $X$ supports a $(1,2)$ Poincaré inequality, i.e., there exists a constant $C_{X}>0$ such that

$$
\int_{X}|v(x)| d \mu(x) \leq C_{X}\left(\int_{X}\left|g_{v}(x)\right|^{2} d \mu(x)\right)^{1 / 2}
$$


for all $v \in N_{0}^{1,2}(\Omega)$.

2). We can modify the proof of Theorem 1.5, similar as that in [22, Proof of Theorem 2 (a)], so that it works for $Y$ being a complete metric space with curvature bounded from above (the so-called CAT(k)-spaces). But then one has to consider mappings into a closed geodesic ball $B$ in $Y$ with radius $R<\frac{\pi}{2 \sqrt{k}}$.

3). The proof shows that Theorem 1.5 holds for the Kuwae-Shioya energy functional if instead we assume $X$ satisfies the $S M C P B G$.

\section{HÖLDER REGUlarity OF HARMONIC MAPPINGS}

In this section, we study the interior regularity of solutions for the Dirichlet problem of Korevaar-Schoen, for which, we term harmonic mappings. We shall prove that harmonic mappings are locally Hölder continuous, provided the metric space $X$ attains certain analytic property and $Y$ is NPC. The proof of Theorem 1.61 ) is based on a combination of the arguments of Jost [45] and Lin [59], while the proof of Theorem 1.6 2) follows closely the approach of Jost [45].

We will need the theory of Dirichlet forms on Hilbert spaces to separate a class of metric spaces, which we name them as metric spaces with property $\mathcal{C}$.

4.1. Dirichlet forms. Recall that a Dirichlet form $\mathcal{E}$ on $L^{2}(X, \mu)$ is a closed nonnegative definite and symmetric bilinear form defined on a dense linear subspace $\mathbb{D}=\mathbb{D}(\mathcal{E})$ of $L^{2}(X, \mu)$, that satisfies the Markovian property

$$
\mathcal{E}(v, v) \leq \mathcal{E}(u, u) \text { for all } u \in \mathbb{D},
$$

where $v=\min \{1, \max \{u, 0\}\}$. A Dirichlet form $\mathcal{E}$ on $L^{2}(X, \mu)$ is said to be strongly local if $\mathcal{E}(u, v)=0$ whenever $u, v \in \mathbb{D}$ with $u$ a constant on a neighborhood of the support of $v$; to be regular if there exists a subset of $D \cap C_{0}(X)$ which is both dense in $C_{0}(X)$ with the uniform norm and in $\mathbb{D}$ with the graph norm $\|\cdot\|_{\mathbb{D}}$ defined by $\|u\|_{\mathbb{D}}=\sqrt{\int_{X} u^{2} d \mu+\mathcal{E}(u, u)}$ for each $u \in \mathbb{D}$.

By the construction of Beurling and Deny [24], each regular strongly local Dirichlet form $\mathcal{E}$ on $L^{2}(X, \mu)$ can be written as

$$
\mathcal{E}(u, v)=\int_{X} d \Gamma(u, v) \text { for all } u, v \in \mathbb{D},
$$

where $\Gamma$ is an $\mathcal{M}(X)$-valued nonnegative definite and symmetric bilinear form defined by the formula

$$
\int_{X} \varphi d \Gamma(u, v)=\frac{1}{2}[\mathcal{E}(u, \varphi v)+\mathcal{E}(v, \varphi u)-\mathcal{E}(u v, \varphi)]
$$

for all $u, v \in \mathbb{D} \cap L^{\infty}(X, \mu)$ and $\varphi \in \mathbb{D} \cap C_{0}(X)$. We call $\Gamma(u, u)$ the Dirichlet energy measure (squared gradient) and $\sqrt{\frac{d \Gamma(u, u)}{d \mu}(x)}$ the length of the gradient of $u$ at $x$.

For a strongly local Dirichlet form $\mathcal{E}$, its energy measure $\Gamma$ is local and satisfies the Leibniz rule and the chain rule. Both $\mathcal{E}(u, v)$ and $\Gamma(u, v)$ can be defined for $u, v \in \mathbb{D}_{\text {loc }}(X)$, the collection of all $u \in L_{\text {loc }}^{2}(X)$ satisfying that for each relatively compact set $K \subset X$, 
there exists a function $w \in \mathbb{D}$ such that $u=w$ almost everywhere on $K$. With this, the intrinsic distance on $X$ associated to $\mathcal{E}$ is defined by

$$
d_{\mathcal{E}}(x, y)=\sup \left\{u(x)-u(y): u \in \mathbb{D}_{\mathrm{loc}}(X) \cap C(X), \Gamma(u, u) \leq \mu\right\} .
$$

Here $\Gamma(u, u) \leq \mu$ means that $\Gamma(u, u)$ is absolutely continuous with respect to $\mu$ and $\frac{d \Gamma(u, u)}{d \mu} \leq 1$ almost everywhere.

Given a Dirichlet form $\mathcal{E}$ on the Hilbert space $L^{2}(X, \mu)$, there exists a unique selfadjoint operator $A$ on $L^{2}(X, \mu)$ with the properties $\mathbb{D}=D\left(A^{1 / 2}\right)$ and

$$
-\mathcal{E}(u, v)=(u, A v)=\int_{X} u(x) A v(x) d \mu(x)
$$

for all $u \in \mathbb{D}$ and $v \in D(A)$.

\subsection{Approximating energy densities with Property $\mathcal{C}$.}

Definition 4.1 (Approximating energy density). Fix an admissible rate function $c: X \times$ $(0, \infty) \rightarrow(0, \infty)$. For each $\varepsilon>0$, we define an approximating energy density $e_{\varepsilon}^{c, Y}$ acting on $u: X \rightarrow Y$ as follows:

$$
e_{\varepsilon}^{c, Y}(u)(x)=\frac{1}{c(x, \varepsilon)} \int_{B(x, \varepsilon)} \frac{d^{2}(u(x), u(y))}{\varepsilon^{2}} d \mu(y) .
$$

We simply write $e_{\varepsilon}^{c}$ when $(Y, d)=(\mathbb{R},|\cdot|)$.

For each $u \in \operatorname{Lip}_{0}(X)$, the space of Lipschitz functions on $X$ with compact support, we introduce the approximating energy $E_{\varepsilon}$ as

$$
E_{\varepsilon}^{c}(u)=\int_{X} e_{\varepsilon}^{c}(u)(x) d \mu(x) .
$$

Definition 4.2 (Property $\mathcal{C}$ ). We say that the approximating energy density $e_{\varepsilon}^{c}$ on a complete metric space $X$ has property $\mathcal{C}$ if the following conditions are satisfied:

(C1): The pointwise limit $E_{0}=\lim _{\varepsilon \rightarrow 0} E_{\varepsilon}^{c}$ exists and induces a regular strongly local Dirichlet forms $\mathcal{E}_{0}$ on $L^{2}(X, \mu)$ via the formula

$$
\mathcal{E}_{0}(u, v)=\lim _{\varepsilon \rightarrow 0} \int_{X} \frac{1}{c(x, \varepsilon)} \int_{B(x, \varepsilon)} \frac{[u(x)-u(y)][v(x)-v(y)]}{\varepsilon^{2}} d \mu(y) d \mu(x) .
$$

Moreover, the intrinsic distance $d_{0}:=d_{\mathcal{E}_{0}}$ associated to $\mathcal{E}_{0}$ is locally bi-Lipschitz equivalent with the original distance $d$ on $X$. In particular, it induces the same topology as the underlying topology on $X$.

(C2): Equip with the intrinsic metric, the space $\left(X, d_{0}, \mu\right)$ becomes a complete locally PI space. That is $\mu$ is a locally doubling measure on $\left(X, d_{0}\right)$ : for each compact set $K \subset\left(X, d_{0}\right)$, there exists a doubling constant $c_{K} \geq 1$ such that

$$
\mu(2 B) \leq c_{K} \mu(B) \quad \text { for each ball } B \text { with } 2 B \subset K \subset\left(X, d_{0}\right),
$$

and that $\left(X, d_{0}, \mu\right)$ supports a local weak $(2,2)$-Poincaré inequality: for each compact set $K \subset\left(X, d_{0}\right)$, there exist $C_{K}>0$ and $\lambda_{K} \geq 1$ such that for each ball 


$$
\begin{aligned}
& B \subset\left(X, d_{0}\right) \text { with } \lambda_{K} B \subset K \text { and all } u \in \mathbb{D}\left(\mathcal{E}_{0}\right), \\
& \qquad \int_{B}\left|u(x)-u_{B}\right|^{2} d \mu(x) \leq C_{K}(\operatorname{diam} B)^{2} \int_{\lambda_{K} B} d \Gamma_{0}(u, u),
\end{aligned}
$$

where $\Gamma_{0}$ is the Dirichlet energy measure corresponding to the Dirichlet form $\mathcal{E}_{0}$.

Remark 4.3. Note that if the approximating energy density $e_{\varepsilon}^{c}$ on a complete metric space $X$ has property $\mathcal{C}$, then it is well-known that $\mathbb{D}\left(\mathcal{E}_{0}\right)=N^{1,2}(X)$ and $\mathbb{D}_{\text {loc }}\left(\mathcal{E}_{0}\right)=N_{\text {loc }}^{1,2}(X)$. Moreover, $\operatorname{Lip}_{0}(X)$ is dense in $\mathbb{D}\left(\mathcal{E}_{0}\right)$; see e.g. [56, Theorem 2.2].

Definition 4.4 (Strong property $\mathcal{C}$ ). We say that the approximating energy density $e_{\varepsilon}^{c, Y}$ between a complete metric space $X$ and an NPC space $Y$ has strong property $\mathcal{C}$ if

- $e_{\varepsilon}^{c}$ has property $\mathcal{C}$;

- for each $u \in K S^{1,2}(X, Y)$, there exists an energy measure $\mu_{u, Y}$ such that $e_{\varepsilon}^{c, Y}(u) d \mu \rightarrow$ $\mu_{u, Y}$ weakly, that is, for each positive function $\eta \in C_{0}(X)$,

$$
\limsup _{\varepsilon \rightarrow 0} \int \eta(x) e_{\varepsilon}^{c, Y}(u)(x) d \mu(x)=\int \eta(x) d \mu_{u, Y}(x)
$$

In particular, if the approximating energy density $e_{\varepsilon}^{c, Y}$ has strong property $\mathcal{C}$, then we have $E(u)(f)=\int_{X} f d \mu_{u, Y}(x)$ for each $u \in K S^{1,2}(X, Y)$ and each $f \in C_{0}(X)$. As an application of Theorem 2.12, we infer that for each compact $K \subset X$, there exists $C_{K}>0$ such that for $u \in K S^{1,2}(X, Y)$,

$$
C_{K}^{-1} \int_{K} g_{u} d \mu \leq \limsup _{\varepsilon \rightarrow 0} \int_{K} e_{\varepsilon}^{c, Y}(u) d \mu \leq C_{K}^{-1} \int_{K} g_{u} d \mu .
$$

Fix an NPC space $Y$, for a large class of metric measure spaces $X$, the KorevaarSchoen approximating energy density $e_{\varepsilon}^{Y}$, i.e. $e_{\varepsilon}^{Y}=e_{\varepsilon}^{c, Y}$ with $c(x, \varepsilon)=\mu(B(x, \varepsilon))$ in Definition 4.1, between $X$ and $Y$, has strong property $\mathcal{C}$.

Example 4.5. Fix an NPC space $Y$. Then on the following $X$, the Korevaar-Schoen approximating energy density $e_{\varepsilon}^{Y}$ between $X$ and $Y$ have strong property $\mathcal{C}$.

1). $X$ is an admissible Riemannian polyhedron.

2). $X$ satisfies the SMCP.

3). $X$ is an $n$-dimensional Lipschitz submanifold of $\mathbb{R}^{N}$.

4). $X$ is $\operatorname{RCD}(K, N)$ in the sense of [27].

Proof. 1). Suppose $M$ is an admissible Riemannian polyhedron. Property (C1) is a direct consequence of [18, Proposition 7.3 and Theorem 9.1] and (C2) follows from [18, Corollary 4.1, Thoerem 5.1]. That $e_{\varepsilon}^{Y}(u) \rightarrow e^{Y}(u)$ is contained in [18, Theorem 9.1].

2). Suppose $X$ satisfies SMCP. Property (C1) follows from [75, Theorem 3.3 and Theorem 6.8]. Property (C2) is a direct consequence of [75, Proposition 4.5 and Theorem 6.4 ] by noticing that the original distance $d$ on $X$ is locally comparable with the intrinsic distance $d_{0}$ induced by the Dirichlet form $\mathcal{E}_{0}$, which is consequence of [75, Proposition 6.6 and Proposition 6.7]. That $e_{\varepsilon}^{Y}(u) \rightarrow e^{Y}(u)$ follows from [75, The proof of Theorem 6.1] by noticing that only a sub-partition lemma is needed for the proof (see also [57, Theorem 4.1]). 
3). The first assertion in property (C1) follows from the subpartition lemma [28, Lemma 3] and the proof of Corollary 5.5 in [75] or alternatively, the proof of Theorem 3.1 in [57]. Property (C2) is clear as being a Lipschitz manifold.

The second claim in property $(\mathrm{C} 1)$ follows by a minor modification of the proofs of Proposition 6.6 and Proposition 6.7 in [75], where one uses the local doubling property of the measure, the local weak (2,2)-Poincaré inequality and [28, Theorem 1]. Alternatively, one can follow the exact proof of Example 4.6 below.

That $e_{\varepsilon}(u) \rightarrow e(u)$ follows from [18, Theorem 1].

4). By [27, Theorem 3.13], the pointwise limt $E_{0}=\lim _{\varepsilon} E_{\varepsilon}$ exists and by [27, Proposition 4.19 and Theorem 4.1], for each $u \in \operatorname{Lip}_{0}(X)$, we have

$$
E_{0}(u)=\int_{X} e_{2}(u)^{2} d \mu=c(d) \int_{X}|d u|^{2} d \mu=c(d) \int_{X}\left|g_{u}\right|^{2} d \mu=c(d) \mathbf{C h}(u),
$$

where $c(d)$ is a constant depending only on the dimension of $X$ and $\mathbf{C h}(u)$ denotes the Cheeger energy of $u$ introduced in [4] (see also [6]). It is well-known that the Cheeger energy function $\mathbf{C h}$ generates a strongly local regular Dirichlet form $\mathcal{E}_{0}$ by the following formula

$$
\mathcal{E}_{0}(u, u)=2 \mathbf{C h}(u, u)
$$

see [5, Section 4.3] or [6]. By [5, Theorem 6.10] the intrinsic distance $d_{0}:=d_{\mathcal{E}_{0}}$ is locally bi-Lipschitz equivalent with the original distance $d$ on $X$. This verifies property $(\mathrm{C} 1)$.

Since $d_{0}$ is locally bi-Lipschitz equivalent to the original metric and $\mu$ is locally doubling on $(X, d)$, it is also locally doubling on $\left(X, d_{0}\right)$. To prove the weak $(2,2)$-Poincaré inequality, first note that $(X, d, \mu)$ supports a weak $(1,2)$-Poincaré inequality (see [66]), and thus by [56, Proposition 2.1 and Proposition 2.2], $\left(X, d_{0}, \mu\right)$ supports a weak $(1,2)$ Poincaré inequality as well. Since the space $\left(X, d_{0}, \mu\right)$ is locally quasiconvex and doubling, by [35, Remark 9.1.19], the weak $(2,2)$-Poincaré inequality (4.5) follows from the weak $(1,2)$-Poincaré inequality. This verifies property (C2).

Note that in our case, we actually have, by [27, Theorem 3.13 and Proposition 4.6], for each $u \in K S^{1,2}(X, Y)$,

$$
e_{\varepsilon}(u) \rightarrow e_{2}(u)^{2}=\mathcal{S}_{2}\left(g_{u}\right)^{2} \quad \text { in } L^{2}(X),
$$

where $\mathcal{S}_{2}$ is a seminorm on $\mathbb{R}^{d}$ defined as in [27, Definition 3.12] and $c(d)>0$ is a constant depending only on the dimension $d$ of $X$. This verifies the additionally required property.

We next verify an example for the Kuwae-Shioya approximating energy density $e_{\varepsilon}^{b, Y}$, i.e. $e_{\varepsilon}^{b}=e_{\varepsilon}^{c, Y}$ with $c(x, \varepsilon)=b(\varepsilon)$ independent of $x$.

Example 4.6. Let $X$ be a complete metric measure space satisfying the SMCPBG and $Y$ an NPC space. Then the Kuwae-Shioya approximating energy density $e_{\varepsilon}^{b, Y}$ between $X$ and $Y$ has strong property $\mathcal{C}$.

Proof. The first assertion in property (C1) follows from [57, Theorem 3.1], where we write $E_{0}^{b}$ instead of $E_{0}$ and $\mathcal{E}_{0}^{b}$ instead of $\mathcal{E}_{0}$. 
We next show the second assertion in property $(\mathrm{C} 1)$, that is, the intrinsic distance $d_{0}$ associated to $\mathcal{E}_{0}^{b}$ satisfies $d_{0} \approx d$ on each compact set $K \subset X$. In particular, it induces the same topology as the underlying topology on $X$. The proof is similar to [75, Proofs of Propositions 6.5 and 6.6].

Claim 1: $d_{0} \geq d$ on $X \times X$.

Indeed, for each $x, z \in X$, each $y \in B(x, \varepsilon)$, the triangle's inequality implies that

$$
\left|\frac{d(x, z)-d(y, z)}{\varepsilon}\right| \leq\left|\frac{d(x, z)-d(y, z)}{d(x, y)}\right| \leq 1 .
$$

Hence, by [57, Theorem 3.1 and Theorem 4.1], for each $z \in X$, the energy measure of the distance function $d_{z}: x \mapsto d(x, z)$ satisfies

$$
\begin{aligned}
\int_{X} \varphi(x) d \Gamma_{0}^{b}\left(d_{z}, d_{z}\right) & =\lim _{\varepsilon \rightarrow 0} \frac{1}{b(\varepsilon)} \int_{X} \varphi(x) \int_{B(x, \varepsilon)}\left(\frac{d(x, z)-d(y, z)}{\varepsilon}\right)^{2} d \mu(y) d \mu(x) \\
& \leq \lim _{\varepsilon \rightarrow 0} \int_{X} \varphi(x)\left(\frac{\mu(B(x, \varepsilon))}{b(\varepsilon)}\right) d \mu(x) \\
& \leq \theta \int_{X} \varphi(x) d \mu(x) .
\end{aligned}
$$

Since $\theta$ can be chosen to be arbitrarily close to 1 , we conclude that

$$
\int_{X} \varphi(x) d \Gamma_{0}^{b}\left(d_{z}, d_{z}\right) \leq \int_{X} \varphi(x) d \mu(x) .
$$

Consequently, $\Gamma_{0}^{b}\left(d_{z}, d_{z}\right) \leq \mu$ and so $d_{0}\left(x, x^{\prime}\right) \geq d_{z}(x)-d_{z}\left(x^{\prime}\right)$ for all $x, x^{\prime}, z \in X$. The claim follows by selecting $z=x^{\prime}$.

Claim 2: for each compact set $K \subset X$, there exists a constant $L$ such that $d_{0} \leq L d$.

Take $u \in \operatorname{Lip}_{0}(X)$ for which $\Gamma_{0}^{b}(u, u) \leq \mu$. Our aim is to show that there exists a constant $L=L\left(K, c_{\mu}\right)$, depending only on $K$ and on the doubling constant $c_{\mu}$, such that for each $z \in K$,

$$
\operatorname{Lip} u(z)=\limsup \sup _{\varepsilon \rightarrow 0} \frac{|u(x)-u(z)|}{\varepsilon} \leq L,
$$

from which we infer that $|u(x)-u(y)| \leq L d(x, y)$ for all $x, y \in K$.

Fix $\varepsilon>0, z \in K$ and

$$
L=L(\varepsilon, z)=\sup \left\{\frac{|u(x)-u(y)|}{\varepsilon}:(x, y) \in B(z, \varepsilon) \times B(z, \varepsilon)\right\} .
$$

Then

$$
|u(x)-u(y)| \leq L \varepsilon \quad \text { for all } x, y \in B(z, \varepsilon) .
$$

Choose $\left(z_{1}, z_{2}\right) \in B(z, \varepsilon) \times B(z, \varepsilon)$ such that

$$
\left|u\left(z_{1}\right)-u\left(z_{2}\right)\right| \geq \frac{1}{2} L \varepsilon>0 .
$$

For $x \in B\left(z_{1}, \frac{1}{8} \varepsilon\right)$ and $y \in B\left(z_{2}, \frac{1}{8} \varepsilon\right)$,

$$
\begin{aligned}
|u(x)-u(y)| & \geq\left|u\left(z_{1}\right)-u\left(z_{2}\right)\right|-\left|u\left(z_{1}\right)-u(x)\right|-\left|u(y)-u\left(z_{2}\right)\right| \\
& \geq \frac{1}{2} L \varepsilon-2 L \varepsilon \frac{1}{8}=\frac{1}{4} L \varepsilon .
\end{aligned}
$$


Then the weak (2,2)-Poincaré inequality from [57, Theorem 4.2] implies that for each $z \in K$ and $\varepsilon<R$,

$$
\int_{B(z, 2 \varepsilon)} f_{B(z, 2 \varepsilon)}\left(\frac{u(x)-u(y)}{\varepsilon}\right)^{2} d \mu(y) d \mu(x) \leq C \mu(B(z, 2 \lambda \varepsilon)),
$$

for some constant $C$ depending on $K$ and $\lambda \geq 1$. Note that

$$
\begin{aligned}
\int_{B(z, 2 \varepsilon)} & \int_{B(z, 2 \varepsilon)}\left(\frac{u(x)-u(y)}{\varepsilon}\right)^{2} d \mu(y) d \mu(x) \\
& \geq \int_{B\left(z_{1}, \varepsilon / 8\right)} \int_{B\left(z_{2}, \varepsilon\right)}\left(\frac{u(x)-u(y)}{\varepsilon}\right)^{2} d \mu(y) d \mu(x) \\
& \geq \frac{1}{16} L^{2} \mu\left(B\left(z_{1}, \varepsilon / 8\right)\right) \mu\left(B\left(z_{2}, \varepsilon / 8\right)\right) .
\end{aligned}
$$

Combining these two inequalities, we obtain

$$
L^{2} \leq 16 C \frac{\mu(B(z, 2 \varepsilon)) \mu(B(z, \lambda \varepsilon))}{\mu\left(B\left(z_{1}, \varepsilon / 8\right)\right) \mu\left(B\left(z_{2}, \varepsilon / 8\right)\right)} \leq \hat{C},
$$

where $\hat{C}$ depends on the compact set $K$ and on the doubling constant of $\mu$. Thus we have shown that for each $z \in K$ and $\varepsilon>0$, there exists $L=L\left(K, c_{\mu}\right)$ such that

$$
|u(x)-u(y)| \leq L \varepsilon
$$

holds for all $x, y \in B(z, \varepsilon)$. In particular, this implies that $\operatorname{Lip} u(z) \leq L$ for all $z \in K$ as desired.

With these understood, property $(\mathrm{C} 2)$ follows from the previous fact and $[57$, Theorem $4.2]$.

Finally, that $e_{\varepsilon}^{b, Y}(u) \rightarrow e^{b, Y}(u)$ follows from [57, Theorem 4.1].

Next, we shall derive some useful consequences for approximating energy density to have (strong) property $\mathcal{C}$.

Lemma 4.7. Assume the approximating energy density $e_{\varepsilon}^{c}$ on a complete metric space $X$ has property $\mathcal{C}$. Then for each $u, v \in \mathbb{D}\left(\mathcal{E}_{0}\right)$ and $\varphi \in \operatorname{Lip}_{0}(X)$, we have

$\lim _{\varepsilon \rightarrow 0} \int_{X} \varphi(x) \frac{1}{c(x, \varepsilon)} \int_{B(x, \varepsilon)} \frac{\left(u(x)-u\left(x^{\prime}\right)\right)\left(v(x)-v\left(x^{\prime}\right)\right)}{\varepsilon^{2}} d \mu\left(x^{\prime}\right) d \mu(x)=\int_{X} \varphi(x) d \Gamma_{0}(u, v)(x)$.

Proof. We first show that for each $u \in \mathbb{D}\left(\mathcal{E}_{0}\right)$ and $\varphi \in \operatorname{Lip}_{0}(X)$,

$$
\lim _{\varepsilon \rightarrow 0} E_{\varepsilon}^{c}(u)(\varphi)=\int_{X} \varphi d \Gamma_{0}(u, u)
$$

or more precisely,

$$
\lim _{\varepsilon \rightarrow 0} \int_{X} \varphi(x) \frac{1}{c(x, \varepsilon)} \int_{B(x, \varepsilon)} \frac{\left(u(x)-u\left(x^{\prime}\right)\right)^{2}}{\varepsilon^{2}} d \mu\left(x^{\prime}\right) d \mu(x)=\int_{X} \varphi(x) d \Gamma_{0}(u, u)(x) .
$$


Indeed, by (4.2) and property (C1), we have

$$
\begin{aligned}
\int_{X} \varphi d \Gamma_{0}(u, u)= & \frac{1}{2}\left[2 \mathcal{E}_{0}(u, \varphi u)-\mathcal{E}_{0}\left(u^{2}, \varphi\right)\right] \\
= & \frac{1}{2}\left[\lim _{\varepsilon \rightarrow 0} \int_{X} \frac{1}{c(x, \varepsilon)} \int_{B(x, \varepsilon)} \frac{2[u(x)-u(y)][\varphi(x) u(x)-\varphi(y) u(y)]}{\varepsilon^{2}}-\right. \\
& \left.\quad-\frac{\left(u^{2}(x)-u^{2}(y)\right)(\varphi(x)-\varphi(y))}{\varepsilon^{2}} \mu(y) d \mu(x)\right] \\
= & \lim _{\varepsilon \rightarrow 0} \int_{X} \varphi(x) \frac{1}{c(x, \varepsilon)} \int_{B(x, \varepsilon)} \frac{(u(x)-u(y))^{2}}{\varepsilon^{2}} d \mu(y) d \mu(x)+ \\
& \frac{1}{2} \lim _{\varepsilon \rightarrow 0} \int_{X} \frac{1}{c(x, \varepsilon)} \int_{B(x, \varepsilon)}(\varphi(y)-\varphi(x)) \frac{(u(x)-u(y))^{2}}{\varepsilon^{2}} d \mu(y) d \mu(x) \\
= & \lim _{\varepsilon \rightarrow 0} E_{\varepsilon}^{c}(u)(\varphi) .
\end{aligned}
$$

Note that

$$
\begin{aligned}
2\left(u(x)-u\left(x^{\prime}\right)\right)\left(v(x)-v\left(x^{\prime}\right)\right)=\left((u+v)(x)-(u+v)\left(x^{\prime}\right)\right)^{2}- & \\
& \left(u(x)-u\left(x^{\prime}\right)\right)^{2}-\left(v(x)-v\left(x^{\prime}\right)\right)^{2} .
\end{aligned}
$$

The claim follows from (4.10) together with the identity

$$
2 \int_{X} \varphi d \Gamma_{0}(u, v)=\int_{X} \varphi d \Gamma_{0}(u+v, u+v)-\int_{X} \varphi d \Gamma_{0}(u, u)-\int_{X} \varphi d \Gamma_{0}(v, v) .
$$

Lemma 4.8. If the approximating energy functional $e_{\varepsilon}^{c, Y}$ between a complete metric space $X$ and an NPC space $Y$ has strong property $\mathcal{C}$, then for each $u \in K S^{1,2}(X, Y), \mu_{u, Y}$ is absolutely continuous with respect to $\mu$.

Proof. Fix a relatively compact domain $K$ in $X$. Suppose $\mu$ is uniformly doubling on balls with radius less than $r_{0}$. It suffices to show that there exists a constant $C$ (depending only on the local uniform doubling constant of $\mu$ on $K$ and on $K$ ) such that for each $B\left(x_{0}, r\right) \subset K, r \leq r_{0}$,

$$
\int_{B\left(x_{0}, r\right)} d \mu_{u, Y}(x) \leq C \int_{B\left(x_{0}, r\right)} g_{u}^{2} d \mu(x) .
$$

Note that (4.11) is isometrically invariant. Indeed, by the proof of Theorem 4.1 in [57], the measure $\mu_{u, Y}$ remains un-changed if we isometrically embeds $Y$ to a Banach space (since the definition of energy is isometrically invariant). The same is true for the right-hand side of (4.11). Thus we may assume that $Y$ is isometrically contained in a Banach space with norm $\|\cdot\|$.

Since

$$
\lim _{\varepsilon \rightarrow 0} \int_{B\left(x_{0}, r\right)} e_{\varepsilon}^{c, Y}(u)(x) d \mu(x)=\int_{B\left(x_{0}, r\right)} d \mu_{u, Y}(x) .
$$

It suffices to give a uniform bound (independent of $\varepsilon$ ) on $\int_{B\left(x_{0}, r\right)} e_{\varepsilon}^{c, Y}(u)(x) d \mu(x)$ in terms of the right-hand side of (4.11). 
Let $g_{u}$ be the minimal 2-weak upper gradient of $u$. Then by $(4.7)$, we have

$$
\int_{B\left(x_{0}, r\right)} e_{\varepsilon}^{c, Y}(u)(x) d \mu(x) \leq C \int_{B\left(x_{0}, r\right)} g_{u}^{2} d \mu(x),
$$

where $C$ is independent of $\varepsilon$. Consequently,

$$
\limsup _{\varepsilon \rightarrow 0} \int_{B\left(x_{0}, r\right)} e_{\varepsilon}^{c, Y}(u)(x) d \mu(x) \leq C \int_{B\left(x_{0}, r\right)} g_{u}^{2} d \mu(x),
$$

from which (4.11) follows.

In particular, if $X$ is uniformly locally doubling and supports a local uniform $(1,2)$ Poincaré inequality, then we would have

$$
d \mu_{u}(x) \leq C(\operatorname{ap}-\operatorname{Lip} u)^{2}(x) d \mu
$$

for all $u \in K S^{1,2}(X, Y)$, where $C$ depends only on the associated data. Recall that for a map $h: X \rightarrow Z$, the approximate pointwise Lipschitz constant of $h$ is defined as

$$
\operatorname{ap}-\operatorname{Liph}(x):=\inf _{A} \limsup _{x^{\prime} \rightarrow x, x^{\prime} \in A \backslash\{x\}} \frac{d\left(h\left(x^{\prime}\right), h(x)\right)}{d\left(x^{\prime}, x\right)},
$$

with the infimum taken over subsets $A \subset X$ having a Lebesgue point of density at $x$. In general, if $x$ is isolated, we let $\operatorname{ap}-\operatorname{Lip} u(x)=0$.

Lemma 4.9. Assume the approximating energy functional $e_{\varepsilon}^{c, Y}$ between a complete metric space $X$ and an $N P C$ space $Y$ has strong property $\mathcal{C}$. Then for each $u \in K S^{1,2}(X, Y)$, each compact set $K \subset\left(X, d_{0}\right)$ with $\lambda_{K} B \subset K$, the following Poincaré inequality holds:

$$
f_{B} \int_{B} d^{2}(u(x), u(y)) d \mu(x) d \mu(y) \leq C_{K}(\operatorname{diam} B)^{2} \int_{\lambda_{K} B} d \mu_{u, Y}(x) .
$$

Proof. Since $e_{\varepsilon}^{c}$ has property $\mathcal{C}$, by [56, Lemma 2.4 and Theorem 2.2], for each $v \in$ $K S^{1,2}(X)$, we have the following $(2,2)$-Poincaré inequality,

$$
\int_{B}\left|v-v_{B}\right|^{2} d \mu(x) \leq C_{K}(\operatorname{diam} B)^{2} \int_{\lambda_{K} B} g_{v}^{2} d \mu
$$

It is well-know that the previous inequality implies that for each $u \in K S^{1,2}(X, V)$, where $V=\left(V,\|\cdot\|_{V}\right)$ is a Banach space, we have

$$
f_{B} \int_{B}\|u(x)-u(y)\|_{V}^{2} d \mu(x) d \mu(y) \leq \hat{C}_{K}(\operatorname{diam} B)^{2} \int_{\lambda_{K} B} g_{u}^{2} d \mu .
$$

For this, see e.g. [35, Proof of Theorem 8.1.42] or [55, Proof of Theorem 3.6]. On the other hand, (4.7) implies

$$
\int_{\lambda_{K} B} g_{u}^{2} d \mu \leq C_{K} \limsup _{\varepsilon \rightarrow 0} \int_{\lambda_{K} B} e_{\varepsilon}^{c, Y}(u)(x) d \mu .
$$

Consequently, for each $u \in K S^{1,2}(X, V)$, we have

$$
f_{B} \int_{B}\|u(x)-u(y)\|_{V}^{2} d \mu(x) d \mu(y) \leq C_{K}(\operatorname{diam} B)^{2} \int_{\lambda_{K} B} d \mu_{u, V} .
$$


Arguing as in the proof of Lemma 4.8 (embedding $Y$ isometrically into some Banach space $V$; say $V=l^{\infty}(Y)$ ), we directly obtain that for each $u \in K S^{1,2}(X, Y)$

$$
f_{B} \int_{B} d^{2}(u(x), u(y)) d \mu(x) d \mu(y) \leq C_{K}(\operatorname{diam} B)^{2} \int_{\lambda_{K} B} d \mu_{u, Y}(x) .
$$

In our following proofs, the metric space $Y$ is typically fixed and we will write $\mu_{u}$, instead of $\mu_{u, Y}$ for notational simplicity.

4.3. Proof of Theorem $\mathbf{1 . 6} \mathbf{1}$ ). We first show that the composition of the distance function with a harmonic mapping is subharmonic in the sense of $[45,8]$. The proof is similar to that of Lemma 5 in [45]; see also [18, Proof of Lemma 10.2].

Proposition 4.10. If $u: X \rightarrow Y$ is harmonic, then for each $y_{0} \in Y$ and each relatively compact open set $U \subset X$, the function $f_{y_{0}}:=d^{2}\left(u(\cdot), y_{0}\right): X \rightarrow \mathbb{R}$ is weakly subharmonic on $U$, i.e., for each positive Lipschitz function $\lambda: X \rightarrow \mathbb{R}$ with $\operatorname{supp}(\lambda) \subset U$,

$$
-\mathcal{E}_{0}\left(\lambda, f_{y_{0}}\right)=\left(\lambda, A f_{y_{0}}\right) \geq 0 \text {. }
$$

Moreover, if $X$ has strong property $C$, then

$$
-\mathcal{E}_{0}\left(\lambda, f_{y_{0}}\right) \geq 2 \int_{X} \lambda d \mu_{u}(x)
$$

Proof. We write $v=d\left(u(\cdot), y_{0}\right)$. Note that both $v$ and $f_{y_{0}}\left(=v^{2}\right)$ are in $\mathbb{D}_{\text {loc }}\left(\mathcal{E}_{0}\right)=N_{\text {loc }}^{1,2}(X)$. Indeed, since the distance function is Lipschitz and $u \in K S_{\text {loc }}^{1,2}(X, Y)$, we easily infer that $v \in K S_{\text {loc }}^{1,2}(X)$. As, under our assumption on $X, K S_{\text {loc }}^{1,2}(X)=N_{\text {loc }}^{1,2}(X)$, we arrive at the desired observation.

Let $\lambda \in \operatorname{Lip}(X), 0 \leq \lambda \leq 1$ be a Lipschitz function with $\operatorname{supp}(\lambda) \subset U$. For each $x \in X$, let $\gamma_{x}:[0,1] \rightarrow Y$ be the constant-speed geodesic in $Y$ from $\gamma_{x}(0)=u(x)$ to $\gamma_{x}(1)=y_{0}$. Define a map $u_{\lambda}: X \rightarrow Y$ by

$$
u_{\lambda}(x)=\gamma_{x}(\lambda(x)), \quad x \in X .
$$

Then $u_{\lambda}=u$ outside $U$. We next compare the energy of $u$ with that of $u_{\lambda}$.

Note that

$$
d\left(u_{\lambda}(x), y_{0}\right) \leq d\left(u(x), y_{0}\right)=v(x)
$$

with $v \in L^{2}(X)$ since $u \in L^{2}(X, Y)$. This implies that $u_{\lambda} \in K S^{1,2}(X, Y)$.

Since $Y$ is NPC, triangle comparison (see [51, Equation (2.1ii)]) gives for $x, x^{\prime} \in X$,

$$
\begin{aligned}
d^{2}\left(u(x), u_{\lambda}\left(x^{\prime}\right)\right) \leq & \left(1-\lambda\left(x^{\prime}\right)\right) d^{2}\left(u(x), u\left(x^{\prime}\right)\right)+\lambda\left(x^{\prime}\right) d^{2}\left(u(x), y_{0}\right) \\
& -\lambda\left(x^{\prime}\right)\left(1-\lambda\left(x^{\prime}\right)\right) d^{2}\left(u\left(x^{\prime}\right), y_{0}\right)
\end{aligned}
$$

and

$$
\begin{aligned}
d^{2}\left(u_{\lambda}(x), u_{\lambda}\left(x^{\prime}\right)\right) \leq & (1-\lambda(x)) d^{2}\left(u(x), u\left(x^{\prime}\right)\right)+\lambda(x) d^{2}\left(u\left(x^{\prime}\right), y_{0}\right) \\
& -\lambda(x)(1-\lambda(x)) d^{2}\left(u(x), y_{0}\right) .
\end{aligned}
$$

Inserting $d\left(u_{\lambda}\left(x^{\prime}\right), y_{0}\right)=\left(1-\lambda\left(x^{\prime}\right)\right) d\left(u\left(x^{\prime}\right), y_{0}\right), d\left(u(\cdot), y_{0}\right)=v$ gives 


$$
\begin{aligned}
& d^{2}\left(u_{\lambda}(x), u_{\lambda}\left(x^{\prime}\right)\right)-d^{2}\left(u(x), u\left(x^{\prime}\right)\right) \\
& \quad \leq-\left[\lambda(x)+\lambda\left(x^{\prime}\right)-\lambda(x) \lambda\left(x^{\prime}\right)\right] d^{2}\left(u(x), u\left(x^{\prime}\right)\right) \\
& \quad-\left(\lambda(x)-\lambda\left(x^{\prime}\right)\right)\left[(1-\lambda(x)) f_{y}(x)-\left(1-\lambda\left(x^{\prime}\right)\right) f_{y}\left(x^{\prime}\right)\right] .
\end{aligned}
$$

Since $\left|\lambda\left(x^{\prime}\right)-\lambda(x)\right| \leq L \cdot \varepsilon$ when $x^{\prime} \in B(x, \varepsilon)$, we have

$$
\lambda(x)+\lambda\left(x^{\prime}\right)-\lambda(x) \lambda\left(x^{\prime}\right)=2 \lambda(x)-\lambda(x)^{2}+O(\varepsilon) .
$$

Now we have by (4.12)

$$
\begin{aligned}
\frac{1}{c(x, \varepsilon)} \int_{B(x, \varepsilon)} \frac{d^{2}\left(u_{\lambda}(x), u_{\lambda}\left(x^{\prime}\right)\right)-d^{2}\left(u(x), u\left(x^{\prime}\right)\right)}{\varepsilon^{2}} d \mu\left(x^{\prime}\right) \\
\leq-\frac{1}{c(x, \varepsilon)} \int_{B(x, \varepsilon)} \frac{\left[2 \lambda(x)-\lambda(x)^{2}+O(\varepsilon)\right] d^{2}\left(u(x), u\left(x^{\prime}\right)\right)}{\varepsilon^{2}} \\
\quad-\frac{\left(\lambda(x)-\lambda\left(x^{\prime}\right)\right)\left[(1-\lambda(x)) f_{y}(x)-\left(1-\lambda\left(x^{\prime}\right)\right) f_{y}\left(x^{\prime}\right)\right]}{\varepsilon^{2}} d \mu\left(x^{\prime}\right) .
\end{aligned}
$$

Multiply by $\eta \in C_{0}(X), 0 \leq \eta \leq 1$, on both side and integrate with respect to $x$, we get

$$
\begin{aligned}
& \int \eta(x) \frac{1}{c(x, \varepsilon)} \int_{B(x, \varepsilon)} \frac{d^{2}\left(u_{\lambda}(x), u_{\lambda}\left(x^{\prime}\right)\right)-d^{2}\left(u(x), u\left(x^{\prime}\right)\right)}{\varepsilon^{2}} d \mu\left(x^{\prime}\right) d \mu(x) \\
& \leq-\int \frac{\eta(x)}{c(x, \varepsilon)} \int_{B(x, \varepsilon)} \frac{\left[2 \lambda(x)-\lambda(x)^{2}+O(\varepsilon)\right] d^{2}\left(u(x), u\left(x^{\prime}\right)\right)}{\varepsilon^{2}} \\
& \quad+\frac{\left(\lambda(x)-\lambda\left(x^{\prime}\right)\right)\left[(1-\lambda(x)) f_{y}(x)-\left(1-\lambda\left(x^{\prime}\right)\right) f_{y}\left(x^{\prime}\right)\right]}{\varepsilon^{2}} d \mu\left(x^{\prime}\right) d \mu(x) .
\end{aligned}
$$

Since $u$ is energy minimizing, the left-hand side of the above inequality is non-negative if we take $\lim \sup _{\varepsilon \rightarrow 0}$. Thus we infer that for $\varepsilon$ sufficiently small

$$
\begin{aligned}
-\kappa(\varepsilon) & +\int \frac{\eta(x)}{c(x, \varepsilon)} \int_{B(x, \varepsilon)} \frac{\left(\lambda(x)-\lambda\left(x^{\prime}\right)\right)\left[(1-\lambda(x)) f_{y}(x)-\left(1-\lambda\left(x^{\prime}\right)\right) f_{y}\left(x^{\prime}\right)\right]}{\varepsilon^{2}} d \mu\left(x^{\prime}\right) d \mu(x) \\
& \leq-\int \frac{\eta(x)}{c(x, \varepsilon)} \int_{B(x, \varepsilon)} \frac{\left[2 \lambda(x)-\lambda(x)^{2}+O(\varepsilon)\right] d^{2}\left(u(x), u\left(x^{\prime}\right)\right)}{\varepsilon^{2}} d \mu\left(x^{\prime}\right) d \mu(x),
\end{aligned}
$$

where $\kappa(\varepsilon)$ is a positive function in $\varepsilon$ that tends to 0 as $\varepsilon \rightarrow 0$. In the above inequality, replace $\lambda$ by $t \lambda$ with $t=\kappa(\varepsilon)^{1 / 2}$, divide by $t$ on both sides and then let $\varepsilon \rightarrow 0$, we obtain

$$
\begin{aligned}
& \limsup _{\varepsilon \rightarrow 0} \int \frac{\eta(x)}{c(x, \varepsilon)} \int_{B(x, \varepsilon)} \frac{\left(\lambda(x)-\lambda\left(x^{\prime}\right)\right)\left(f_{y_{0}}(x)-f_{y_{0}}\left(x^{\prime}\right)\right)}{\varepsilon^{2}} d \mu\left(x^{\prime}\right) d \mu(x) \\
& \leq \limsup _{\varepsilon \rightarrow 0}-\int \frac{\eta(x)}{c(x, \varepsilon)} \int_{B(x, \varepsilon)} \frac{2 \lambda(x) d^{2}\left(u(x), u\left(x^{\prime}\right)\right)}{\varepsilon^{2}} d \mu\left(x^{\prime}\right) d \mu(x) \leq 0 .
\end{aligned}
$$

Together with Lemma 4.7, we deduce that

$$
\int_{X} \eta d \Gamma_{0}\left(\lambda, f_{y_{0}}\right) \leq 0 .
$$


In particular, we have $\left(\lambda, A f_{y_{0}}\right) \geq 0$ for all positive Lipschitz function $\lambda$ with $\operatorname{supp}(\lambda) \subset U$. If $X$ has strong property $\mathcal{C}$, then we get from the previous inequality that

$$
\int_{X} \eta d \Gamma_{0}\left(\lambda, f_{y_{0}}\right) \leq-2 \int_{X} \eta(x) \lambda(x) d \mu_{u}(x) .
$$

We next point out that as in the setting of Riemannian manifolds, subharmonic functions are locally bounded.

Lemma 4.11. Every positive weakly subharmonic function $v: X \rightarrow \mathbb{R}$ is locally bounded.

Proof. By [8, Theorem 5.4], for every $p>0$, there exists a positive constant $c_{p}$ such that

$$
\sup _{B_{0}(x, r / 2)} v \leq c_{p}\left(f_{B_{0}(x, r)} v^{p} d \mu(x)\right)^{1 / p} .
$$

The claim follows by taking $p=2$.

Our proof of interior Hölder regularity follows the general approach of Lin [59, Proof of Theorem 3.1]. For this, we need the following key covering type lemma, which generalizes [59, Lemma 3.5] via a similar idea to the current setting.

From now on (till the end of this section), we assume that $Y$ is a locally doubling NPC space and the approximating energy functional $e_{\varepsilon}^{c, Y}$ between $X$ and $Y$ has property $\mathcal{C}$. Recall that a metric space $Y$ is doubling with constant $M, M \in \mathbb{N}$, if for each ball $B(x, r)$, every $r / 2$-separated subset of $B(x, r)$ has at most $M$ points. $Y$ is locally doubling if each compact subset $K$ of $Y$ is doubling with some constant $C_{K}$.

Lemma 4.12. Let $u: X \rightarrow Y$ be given as in Proposition 4.10. We further assume that $\mu$ is doubling on $X$ with constant $c_{d}, X$ supports a weak $(2,2)$-Poincaré inequality with constant $C_{P}$ and $Y$ is $M$-doubling. Suppose $\kappa=\operatorname{diam} u\left(B\left(x_{0}, r\right)\right) \in\left[\kappa_{1}, \kappa_{0}\right]$. There exists some $\varepsilon_{0}>0$, depending only on $\kappa_{0}, \kappa_{1}$ and $c_{d}, C_{P}$ and $M$ such that if $u\left(B\left(x_{0}, r\right)\right)$ is covered by $k$ balls $B_{1}, \ldots, B_{k}$ of radius $\varepsilon \leq \varepsilon_{0}$, then $u\left(B\left(x_{0}, r / 2\right)\right)$ can be covered by at most $k-1$ balls among $B_{1}, \ldots, B_{k}$.

Proof. For $i=1,2, \ldots, k$, we take $x_{i} \in B\left(x_{0}, r\right)$ such that $B_{i} \subset B\left(p_{i}, 2 \varepsilon\right)$, where $p_{i}=u\left(x_{i}\right)$. Since $\varepsilon \leq \varepsilon_{0} \leq \kappa / 16$, the balls $B\left(p_{i}, \kappa / 8\right)$ covers $u\left(B\left(x_{0}, r\right)\right)$. Since $u\left(B\left(x_{0}, r\right)\right)$ has diameter $\kappa$, every $p_{i}$ belongs to a closed ball $\bar{B}^{\prime}$ of radius $\kappa$ in $Y$.

Let $k^{\prime}$ be the maximal number of points in the ball such that the distance is at least $\kappa / 8$ apart. Since $Y$ is doubling, $k^{\prime} \leq C_{Y}$ for some constant $C_{Y}$ depending only on the doubling constant $M$ of $Y$. Thus, we may assume that $B\left(p_{i}, \kappa / 4\right), i=1,2, \ldots, k^{\prime}$ covers $u\left(B\left(x_{0}, r\right)\right)$. It follows that for at least one of those $p_{i}$, say for $p_{1}$,

$$
\mu\left(u^{-1}\left(B\left(p_{1}, \kappa / 4\right)\right) \cap B\left(x_{0}, r / 2\right)\right) \geq \frac{1}{k^{\prime}} \mu\left(B\left(x_{0}, r / 2\right)\right)
$$

Consider the auxiliary function $f_{p_{1}}(x):=\frac{1}{\kappa^{2}} d^{2}\left(u(x), p_{1}\right)$. It is clear that

$$
\tau:=\sup _{x \in B\left(x_{0}, r\right)} f_{p_{1}}(x) \leq \frac{1}{\kappa^{2}}\left(\operatorname{diam} u\left(B\left(x_{0}, r\right)\right)\right)^{2} \leq 1 .
$$


By the triangle inequality, and since diam $u\left(B\left(x_{0}, r\right)\right)=\kappa$, there exists some $\hat{x} \in B\left(x_{0}, r\right)$ with $f_{p_{1}}(\hat{x}) \geq \kappa / 2$, and so $\tau \geq \frac{1}{4}$. Since the function $h(x):=\tau-f_{p_{1}}(x) \geq 0$ on $B\left(x_{0}, r\right)$ and $h(x) \geq \frac{1}{8}$ on $u^{-1}\left(B\left(p_{1}, \kappa / 4\right)\right)$. Since $f_{p_{1}}$ is weakly subharmonic, $h$ is weakly supharmonic and so by [45, Lemma 6$]$,

$$
\inf _{x \in B\left(x_{0}, r / 2\right)} h(x) \geq c_{1} f_{B\left(x_{0}, r\right)} h(x) d \mu(x) \geq \frac{c_{1}}{k^{\prime}} \frac{\mu\left(B\left(x_{0}, r / 2\right)\right)}{\mu\left(B\left(x_{0}, r\right)\right)} \geq c_{0} .
$$

We next claim that for sufficiently small $\varepsilon$, we cannot have $u\left(B\left(x_{0}, \frac{r}{2}\right)\right) \cap B\left(p_{i}, 2 \varepsilon\right) \neq \emptyset$ for all $i=1, \ldots, k$. Indeed, let $\tilde{x} \in B\left(x_{0}, r\right)$ be such that $\tau=\frac{1}{\kappa^{2}} d^{2}\left(u(\tilde{x}), p_{1}\right)$. since the balls $B\left(p_{i}, 2 \varepsilon\right)$ cover $u\left(B\left(x_{0}, r\right)\right)$, we find some $p_{i}$ with $d\left(u(\tilde{x}), p_{i}\right) \leq 2 \varepsilon$. Thus, if $u\left(B\left(x_{0}, \frac{r}{2}\right)\right) \cap B\left(p_{i}, 2 \varepsilon\right) \neq \emptyset$ for all $i=1, \ldots, k$, we would have $d\left(u\left(x_{1}\right), u(\tilde{x})\right) \leq 4 \varepsilon$ for some $x_{1} \in B\left(x_{0}, r / 2\right)$ and so

$$
\begin{aligned}
\inf _{x \in B\left(x_{0}, r / 2\right)} h(x) & \leq h\left(x_{1}\right)=\tau-\frac{1}{\kappa^{2}} d^{2}\left(u\left(x_{1}\right), p_{1}\right) \\
& \leq \tau-\frac{1}{\kappa^{2}}\left(d^{2}\left(u(\tilde{x}), p_{1}\right)+d^{2}\left(u(\tilde{x}), u\left(x_{1}\right)\right)-2 d\left(u(\tilde{x}), p_{1}\right) d\left(u(\tilde{x}), u\left(x_{1}\right)\right)\right) \\
& \leq \frac{16 \varepsilon \sqrt{\tau}}{\kappa},
\end{aligned}
$$

which contradicts (4.14) if $\varepsilon_{0}$ is sufficiently small, quantitatively.

With the help of Lemma 4.12, the proof of interior Hölder regularity can be deduced by a similar argument as in Lin [59, Proof of Theorem 3.1].

Proposition 4.13. Let $u: X \rightarrow Y$ be given as in Proposition 4.10, then $u$ is locally Hölder continuous.

Proof. Since the issue is local, we may assume that $\mu$ is a global doubling measure on $X$ with doubling constant $c_{d}$ and $X$ supports a weak $(2,2)$-Poincaré inequality with constant $C_{P}$ and $Y$ is $M$-doubling. In below, we refer $c_{d}, C_{P}$ and $M$ as the data of $X$ and $Y$. Under these assumptions, we first show that there exists $\delta_{0}$, depending only on the data of $X$ and $Y$ such that if $B\left(x_{0}, 2\right) \subset \subset \Omega$, then diam $u\left(B\left(x_{0}, 1\right)\right)=2$ implies that $\operatorname{diam} u\left(B\left(x_{0}, \delta\right)\right) \leq 1$. For notational simplicity, we write $B_{t}=B\left(x_{0}, t\right)$ for $t>0$.

Let $\varepsilon_{0}$ be given as in Lemma 4.12. Since diam $u\left(B_{1}\right)=2$, we can cover $u\left(B_{1}\right)$ by $k$ balls of radius $\varepsilon_{0}$ in $N$, where $k$ depends only on the data of $X$ and $Y$. By Lemma 4.12, $u\left(B_{2^{-1}}\right)$ can be covered by at most $k-1$ balls of radius $\varepsilon_{0}$. If $\operatorname{diam} u\left(B_{2^{-1}}\right)>1$, we may repeat the above arguments with $2^{-1}$ in place of 1 and $k-1$ in place of $k$ to conclude that $u\left(B_{2^{-2}}\right)$ can be covered by at most $k-2$ balls. It follows that there is some $k_{0} \leq k$ such that $\operatorname{diam} u\left(B_{2^{-k_{0}}}\right) \leq 1$. In particular, we may set $\delta=2^{-k_{0}}$ for the claim. 
Consider now $B\left(x_{0}, r\right)$ with $B\left(x_{0}, 2 r\right) \subset \subset \Omega$. Let $\kappa=\operatorname{diam} u\left(B\left(x_{0}, r\right)\right)$. Denote by $\hat{X}=\left(X, r^{-1} d, \mu\right)$ and $Y_{\kappa}=\left(Y, 2 \kappa^{-1} d\right)$. Then for each $v \in \operatorname{Lip}_{0}(X)$,

$$
\begin{aligned}
\hat{E}_{\varepsilon}(v) & =\int_{X} \frac{1}{c(x, r \varepsilon)} \int_{\hat{B}(x, \varepsilon)} \frac{|v(x)-v(y)|^{2}}{\varepsilon^{2}} d \mu(y) d \mu(x) \\
& =\int_{X} \frac{1}{c(x, r \varepsilon)} \int_{B(x, r \varepsilon)} \frac{|v(x)-v(y)|^{2}}{\varepsilon^{2}} d \mu(y) d \mu(x) \\
& =r^{2} E_{r \varepsilon}(v) .
\end{aligned}
$$

Thus, $\lim _{\varepsilon \rightarrow 0} \hat{E}_{\varepsilon}(u)=r^{2} E_{0}(u)$ exists and induces a regular strongly local Dirichlet form $\hat{\mathcal{E}}_{0}=r^{2} \mathcal{E}_{0}$. Let $\hat{\Gamma}_{0}$ be the energy measure associated to the Dirichlet form $\hat{\mathcal{E}}_{0}$. Then $d \hat{\Gamma}_{0}=r^{2} d \Gamma_{0}$.

We next show that $\hat{X}$ and $Y_{\kappa}$ have the same data as that of $X$ and $Y$. Indeed, for each $\hat{B}(x, s) \subset \hat{X}$,

$$
\mu(\hat{B}(x, 2 s))=\mu(B(x, 2 s r)) \leq c_{d} \mu(B(x, s r))=c_{d} \mu(\hat{B}(x, s))
$$

and

$$
\begin{aligned}
\int_{\hat{B}(x, s)}\left|v(y)-v_{\hat{B}(x, s)}\right|^{2} d \mu(y) & =\int_{B(x, s r)}\left|v-v_{B(x, s r)}\right|^{2} d \mu \\
& \leq C_{P}(\operatorname{diam} B(x, s r))^{2} \int_{\lambda B(x, s r)} d \Gamma_{0}(v, v) \\
& =C_{p}(\operatorname{diam} \hat{B}(x, s))^{2} \int_{\lambda \hat{B}(x, s)} d \hat{\Gamma}_{0}(v, v)
\end{aligned}
$$

That $Y_{\kappa}$ is $M$-doubling is clear.

We now regard our harmonic mapping $u: X \rightarrow Y$ as a mapping $\hat{u}: \hat{X} \rightarrow\left(Y, 2 \kappa^{-1} d\right)$. Then a simple computation implies

$$
\hat{E}\left(u_{r}\right)=\inf _{v \in K S_{\phi}^{1,2}(\Omega, Y)} \hat{E}(v)
$$

which means that $\hat{u}: \hat{X} \rightarrow Y_{\kappa}$ is harmonic. Moreover,

$$
\operatorname{diam}_{Y_{\kappa}} \hat{u}\left(\hat{B}\left(x_{0}, 1\right)\right)=2 k^{-1} \operatorname{diam} u\left(B\left(x_{0}, r\right)\right)=2 .
$$

Thus, we may apply Lemma 4.12 to find some $\delta$, depending only on the data of $\hat{X}$ and $Y_{\kappa}$ (and hence on the data of $X$ and $\left.Y\right)$, such that $\operatorname{diam}_{Y_{\kappa}} \hat{u}\left(\hat{B}\left(x_{0}, \delta\right)\right) \leq 1$. That is, $2 k^{-1} \operatorname{diam} u\left(B\left(x_{0}, \delta r\right)\right) \leq 1$, or equivalently, $\operatorname{diam} u\left(B\left(x_{0}, \delta r\right)\right) \leq \frac{1}{2} \operatorname{diam} u\left(B\left(x_{0}, r\right)\right)$. A standard iteration then gives the desired Hölder continuity.

Proof of Theorem 1.6 1). This follows from Proposition 4.13.

4.4. Proof of Theorem 1.6 2). We will now follow the approach of Jost [45] to prove the interior regularity for harmonic mappings from metric spaces with property $\mathcal{C}$ to NPC spaces. Throughout this section, we consider everything in the metric space $\left(X, d_{0}\right)$. For instance, when we say $B\left(x_{0}, R\right) \subset X$ is a ball, we mean that $B(x, R)$ is a ball in $\left(X, d_{0}\right)$. 
If $B\left(x_{0}, R\right) \subset X$ is a ball with fixed center $x_{0}$, we write

$$
v_{+, R}:=\sup _{x \in B\left(x_{0}, R\right)} v(x), v_{-, R}:=\inf _{x \in B\left(x_{0}, R\right)} v(x) \text { and } v_{R}:=f_{B\left(x_{0}, R\right)} v(x) d \mu(x) .
$$

The following lemma is a variant of [45, Lemma 8].

Lemma 4.14. Suppose the approximating energy functional $e_{\varepsilon}^{c, Y}$ between a complete metric space $X$ and an $N P C$ space $Y$ has strong property $\mathcal{C}$. Let $u: X \rightarrow Y$ be a harmonic mapping. Fix $y_{0} \in Y$ and $B\left(x_{0}, 4 R\right) \subset \subset X$. Then

$$
\frac{R^{2}}{\mu\left(x_{0}, R\right)} \int_{B\left(x_{0}, R\right)} d \mu_{u}(x) \leq c\left(v_{+, 4 R}-v_{+, R}\right),
$$

where $v(x)=f_{y_{0}}(x)=d^{2}\left(u(x), y_{0}\right)$.

Proof. Note that by Proposition 4.10 we have $2 \int \lambda(x) d \mu_{u}(x) \leq-\mathcal{E}_{0}(\lambda, v)$ for each positive Lipschitz function $\lambda$ with compact support.

Fix $B\left(x_{0}, 4 R\right) \subset \subset X$. Let $G^{R}\left(x_{0}, x\right)$ be the singular Green function on $B\left(x_{0}, R\right)$ relative to $B\left(x_{0}, 2 R\right)$, that is, $G^{R}\left(x_{0}, \cdot\right) \in \mathbb{D}\left(\mathcal{E}_{0}, B\left(x_{0}, 2 R\right)\right)$ (closure of $\mathbb{D}\left(\mathcal{E}_{0}\right) \cap C_{0}\left(B\left(x_{0}, 2 R\right)\right)$ ) and it is the unique solution of

$$
\left(-\int_{B\left(x_{0}, 2 R\right)} \varphi(x) A G^{R}\left(x_{0}, x\right) d \mu(x)=\right) \mathcal{E}_{0}\left(\varphi, G^{R}\left(x_{0}, \cdot\right)\right)=f_{B\left(x_{0}, R\right)} \varphi(x) d \mu(x)
$$

for all $\varphi \in \mathbb{D}\left(\mathcal{E}_{0}\right)$ with $\operatorname{supp}(\varphi) \subset B\left(x_{0}, 2 R\right)$; see [8, Section 6] for the existence and basic properties of this Green function. Set

$$
w^{R}(x):=\frac{\mu\left(B\left(x_{0}, R\right)\right)}{R^{2}} G^{R}\left(x_{0}, x\right) \in \mathbb{D}\left(\mathcal{E}_{0}, B\left(x_{0}, 2 R\right)\right) .
$$

Then

$$
\mathcal{E}_{0}\left(\varphi, w^{R}\right)=\frac{1}{R^{2}} \int_{B\left(x_{0}, R\right)} \varphi(x) d \mu(x)
$$

for all $\varphi \in \mathbb{D}\left(\mathcal{E}_{0}\right)$ with $\operatorname{supp}(\varphi) \subset B\left(x_{0}, 2 R\right)$. Furthermore, by the estimates for $G^{R}[8$, Theorem 6.1], we have

$$
\begin{array}{ll}
0 \leq w^{R} \leq \gamma_{1} & \text { in } B\left(x_{0}, 2 R\right) \\
w^{R} \geq \gamma_{2}>0 & \text { in } B\left(x_{0}, R\right)
\end{array}
$$

for some structural constants $\gamma_{1}, \gamma_{2}$ that does not depend on $R$.

Set $z(x):=v(x)-v_{+, 4 R}$. Then we have

$$
\begin{aligned}
2 \int_{B\left(x_{0}, 2 R\right)}\left(w^{R}(x)\right)^{2} d \mu_{u}(x) & \leq-\mathcal{E}_{0}\left(\left(w^{R}\right)^{2}, z\right) \\
& =-2 \mathcal{E}_{0}\left(w^{R}, w^{R} z\right)+2 \int z d \Gamma_{0}\left(w^{R}, w^{R}\right) \\
& \leq-2 \mathcal{E}_{0}\left(w^{R}, w^{R} z\right) \quad \text { since } z \leq 0 .
\end{aligned}
$$


From (4.15), (4.16), (4.17) and [45, Corollary 1], we obtain

$$
\begin{aligned}
\int_{B\left(x_{0}, R\right)} d \mu_{u}(x) & \leq c_{1} \int_{B\left(x_{0}, 2 R\right)}\left(w^{R}(x)\right)^{2} d \mu_{u}(x) \\
& \leq-\frac{c_{1}}{R^{2}} \int_{B\left(x_{0}, R\right)} w^{R}(x) z(x) d \mu(x) \\
& \leq \frac{c_{2}}{R^{2}} \int_{B\left(x_{0}, R\right)} v_{+, 4 R}-v(x) d \mu(x) \\
& =\frac{c_{2} \mu\left(B\left(x_{0}, R\right)\right)}{R^{2}}\left(v_{+, 4 R}-v_{R}\right) \\
& \leq \frac{c_{3} \mu\left(B\left(x_{0}, R\right)\right)}{R^{2}}\left(v_{+, 4 R}-v_{+, R}\right) .
\end{aligned}
$$

Proof of Theorem 1.6 2). With Lemma 4.9 and Lemma 4.14 at hand, the proof of [45, Theorem, Section 6] works with minor changes in our setting.

For each $\rho>0$, we set

$$
\omega(\rho):=\sup _{x \in B\left(x_{0}, \rho\right)} d^{2}(u(x), p)=v_{p,+, \rho}
$$

Our aim is to show that for each $p$ in the convex hull of $u\left(B\left(x_{0}, \delta R\right)\right)$, where $\delta$ is a fixed constant, and for all $\rho<\frac{R}{2}$ sufficiently small,

$$
\omega(\rho) \leq c\left(\frac{\rho}{R}\right)^{\alpha} \omega(R)
$$

for some constant $c>0$ and some $\alpha \in(0,1)$.

Before turn to the proof of the claim, we observe the claim implies the Hölder continuity via a standard argument as follows. Take $p=\bar{u}_{\rho}$, the mean value (or the center of mass) of $u$ on $B\left(x_{0}, \rho\right)$, then

$$
\omega(\rho)^{1 / 2} \leq \operatorname{osc}_{B\left(x_{0}, \rho\right)} u \leq 2 \omega(\rho)^{1 / 2}
$$

from which the Hölder continuity follows.

Since the issue is isometrically invariant, we may assume that $Y$ is (isometrically) contained in some Banach space $V$ with norm $\|\cdot\|$. Set $\bar{u}_{R}$ be the mean value of $u$ on $B\left(x_{0}, R\right)$ and $v_{p}(x):=\|u(x)-p\|^{2}$. We shall apply [45, Lemma 7] to the function $v_{\bar{u}_{\frac{R}{4}}}$. Choose $\varepsilon=\frac{1}{8}$ and $R^{\prime} \in\left[\varepsilon^{m} R, \frac{R}{4}\right]$. Since $X$ has strong property C, we have by Lemma 4.9 and Lemma 4.14 that

$$
\begin{aligned}
v_{R^{\prime}} & =f_{B\left(x_{0}, R^{\prime}\right)}\left\|u(x)-\bar{u}_{\frac{R}{4}}\right\|^{2} d \mu(x) \leq C_{0} f_{B\left(x_{0}, R / 4\right)}\left\|u(x)-\bar{u}_{\frac{R}{4}}\right\|^{2} d \mu(x) \\
& \leq \frac{C_{1} R^{2}}{\mu\left(B\left(x_{0}, R\right)\right)} \int_{B\left(x_{0}, \lambda R\right)} d \mu_{u}(x) \leq C_{2}\left(v_{p,+, \lambda R}-v_{p,+, \lambda R / 4}\right)
\end{aligned}
$$


Combining this estimate with [45, Lemma 7], we get for each $p$ in the convex hull of $u\left(B\left(x_{0}, \delta R\right)\right), \delta=\varepsilon^{m}$, we have

$$
\begin{aligned}
\sup _{x \in B\left(x_{0}, \delta R\right)}\|u(x)-p\|^{2} & \leq 4 \sup _{x \in B\left(x_{0}, \delta R\right)}\left\|u(x)-\bar{u}_{\frac{R}{4}}\right\|^{2} \\
& \leq 4 \varepsilon^{2} \sup _{x \in B\left(x_{0}, R\right)}\left\|u(x)-\bar{u}_{\frac{R}{4}}\right\|^{2}+C_{3}\left(v_{p,+, \lambda R}-v_{p,+, \lambda R / 4}\right) \\
& \leq 16 \varepsilon^{2} \sup _{x \in B\left(x_{0}, R\right)}\left\|u(x)-\bar{u}_{\frac{R}{4}}\right\|^{2}+C_{3}\left(v_{p,+, \lambda R}-v_{p,+, \delta R}\right) .
\end{aligned}
$$

Since

$$
\sup _{x \in B\left(x_{0}, R\right)}\|u(x)-p\|^{2} \leq \sup _{x \in B\left(x_{0}, \lambda R\right)}\|u(x)-p\|^{2}=\omega(\lambda R)
$$

we have

$$
\left(1+C_{3}\right) \omega(\delta R) \leq\left(\frac{1}{64}+C_{3}\right) \omega(\lambda R)
$$

A simple iteration then gives our desired estimate

$$
\omega(\rho) \leq c\left(\frac{\rho}{R}\right)^{\alpha} \omega(R)
$$

for some constant $c>0$ and some $\alpha \in(0,1)$.

\section{A Liouille type TheOREM FOR HARMONiC MAPPingS}

5.1. Composition with distance function. We first show that the composition of the distance function with a harmonic mapping $u$ is "almost" weakly subharmonic. The proof is similar to that used in Proposition 4.10, relying on the idea of Jost [45].

Lemma 5.1. If $u: X \rightarrow Y$ is harmonic, then for each $y_{0} \in Y$, the function $v=$ $d\left(u(\cdot), y_{0}\right): X \rightarrow \mathbb{R}$ satisfies that for each positive $\lambda \in N^{1,2}(X) \cap L^{\infty}(X)$ with compact support,

$$
-\mathcal{E}_{0}(\lambda v, v) \geq 0
$$

Proof. Let $\lambda \in \operatorname{Lip}_{0}(X), 0 \leq \lambda \leq 1$ be a Lipschitz function with $\operatorname{supp}(\lambda) \subset U \subset \subset X$. By standard approximation, it suffices to prove (5.1) holds for all such $\lambda$. For each $x \in X$, let $\gamma_{x}:[0,1] \rightarrow Y$ be the constant-speed geodesic in $Y$ from $\gamma_{x}(0)=u(x)$ to $\gamma_{x}(1)=y_{0}$. Define a map $u_{\lambda}: X \rightarrow Y$ by

$$
u_{\lambda}(x)=\gamma_{x}(\lambda(x)), \quad x \in X .
$$

Then $u_{\lambda}=u$ outside $U$. We next compare the energy of $u$ with that of $u_{\lambda}$.

Note that

$$
d\left(u_{\lambda}(x), y_{0}\right) \leq d\left(u(x), y_{0}\right)=v(x)
$$

with $v \in L^{2}(X)$ since $u \in L^{2}(X, Y)$. This implies that $u_{\lambda} \in K S^{1,2}(X, Y)$.

Since $Y$ is NPC, triangle comparison (see [51, Equation (2.1ii)]) gives for $x, x^{\prime} \in X$,

$$
\begin{aligned}
d^{2}\left(u(x), u_{\lambda}\left(x^{\prime}\right)\right) \leq & (1-\lambda(x)) d^{2}\left(u(x), u\left(x^{\prime}\right)\right)+\lambda\left(x^{\prime}\right) d^{2}\left(u(x), y_{0}\right) \\
& -\lambda\left(x^{\prime}\right)\left(1-\lambda\left(x^{\prime}\right)\right) d^{2}\left(u\left(x^{\prime}\right), y_{0}\right)
\end{aligned}
$$


and

$$
\begin{aligned}
d^{2}\left(u_{\lambda}(x), u_{\lambda}\left(x^{\prime}\right)\right) \leq & (1-\lambda(x)) d^{2}\left(u(x), u\left(x^{\prime}\right)\right)+\lambda(x) d^{2}\left(u\left(x^{\prime}\right), y_{0}\right) \\
& -\lambda(x)(1-\lambda(x)) d^{2}\left(u(x), y_{0}\right) .
\end{aligned}
$$

Inserting $d\left(u_{\lambda}\left(x^{\prime}\right), y_{0}\right)=\left(1-\lambda\left(x^{\prime}\right)\right) d\left(u\left(x^{\prime}\right), y_{0}\right), d\left(u(\cdot), y_{0}\right)=v$ gives

$$
\begin{aligned}
& d^{2}\left(u_{\lambda}(x), u_{\lambda}\left(x^{\prime}\right)\right)-d^{2}\left(u(x), u\left(x^{\prime}\right)\right) \\
& \quad \leq-\left[\lambda(x)+\lambda\left(x^{\prime}\right)-\lambda(x) \lambda\left(x^{\prime}\right)\right]\left[d^{2}\left(u(x), u\left(x^{\prime}\right)\right)-\left(v(x)-v\left(x^{\prime}\right)\right)^{2}\right] \\
& \quad-2\left(v(x)-v\left(x^{\prime}\right)\right)\left[\lambda(x) v(x)-\lambda\left(x^{\prime}\right) v\left(x^{\prime}\right)\right]+\left[\lambda(x) v(x)-\lambda\left(x^{\prime}\right) v\left(x^{\prime}\right)\right]^{2} .
\end{aligned}
$$

Since $\left|\lambda\left(x^{\prime}\right)-\lambda(x)\right| \leq L \cdot \varepsilon$ when $x^{\prime} \in B(x, \varepsilon)$, we have

$$
\lambda(x)+\lambda\left(x^{\prime}\right)-\lambda(x) \lambda\left(x^{\prime}\right)=2 \lambda(x)-\lambda(x)^{2}+O(\varepsilon) .
$$

Now we have by $(5.2)$

$$
\begin{aligned}
\frac{1}{c(x, \varepsilon)} \int_{B(x, \varepsilon)} \frac{d^{2}\left(u_{\lambda}(x), u_{\lambda}\left(x^{\prime}\right)\right)-d^{2}\left(u(x), u\left(x^{\prime}\right)\right)}{\varepsilon^{2}} d \mu\left(x^{\prime}\right) \\
\leq-\frac{1}{c(x, \varepsilon)} \int_{B(x, \varepsilon)} \frac{\left[2 \lambda(x)-\lambda(x)^{2}+O(\varepsilon)\right]\left[d^{2}\left(u(x), u\left(x^{\prime}\right)\right)-\left(v(x)-v\left(x^{\prime}\right)\right)^{2}\right]}{\varepsilon^{2}} \\
\quad+\frac{2\left(v(x)-v\left(x^{\prime}\right)\right)\left[\lambda(x) v(x)-\lambda\left(x^{\prime}\right) v\left(x^{\prime}\right)\right]+\left[\lambda(x) v(x)-\lambda\left(x^{\prime}\right) v\left(x^{\prime}\right)\right]^{2}}{\varepsilon^{2}} d \mu\left(x^{\prime}\right) .
\end{aligned}
$$

Multiply by $\eta$ on both side and integrate with respect to $x$, we get

$$
\begin{aligned}
\int \frac{\eta(x)}{c(x, \varepsilon)} & \int_{B(x, \varepsilon)} \frac{d^{2}\left(u_{\lambda}(x), u_{\lambda}\left(x^{\prime}\right)\right)-d^{2}\left(u(x), u\left(x^{\prime}\right)\right)}{\varepsilon^{2}} d \mu\left(x^{\prime}\right) d \mu(x) \\
\leq & -\int \frac{\eta(x)}{c(x, \varepsilon)} \int_{B(x, \varepsilon)} \frac{\left[2 \lambda(x)-\lambda(x)^{2}+O(\varepsilon)\right]\left[d^{2}\left(u(x), u\left(x^{\prime}\right)\right)-\left(v(x)-v\left(x^{\prime}\right)\right)^{2}\right]}{\varepsilon^{2}} \\
& +\frac{2\left(v(x)-v\left(x^{\prime}\right)\right)\left[\lambda(x) v(x)-\lambda\left(x^{\prime}\right) v\left(x^{\prime}\right)\right]+\left[\lambda(x) v(x)-\lambda\left(x^{\prime}\right) v\left(x^{\prime}\right)\right]^{2}}{\varepsilon^{2}} d \mu\left(x^{\prime}\right) d \mu(x) .
\end{aligned}
$$

Since $u$ is energy minimizing, the left-hand side of the above inequality is non-negative if we take $\lim \sup _{\varepsilon \rightarrow 0}$. Thus, we infer that

$$
\begin{aligned}
-\kappa(\varepsilon) & +\int \frac{\eta(x)}{c(x, \varepsilon)} \int_{B(x, \varepsilon)} \frac{\left[2 \lambda(x)-\lambda(x)^{2}\right]\left[d^{2}\left(u(x), u\left(x^{\prime}\right)\right)-\left(v(x)-v\left(x^{\prime}\right)\right)^{2}\right]}{\varepsilon^{2}} d \mu\left(x^{\prime}\right) d \mu(x) \\
& \leq-\int \frac{\eta(x)}{c(x, \varepsilon)} \int_{B(x, \varepsilon)} \frac{2\left(v(x)-v\left(x^{\prime}\right)\right)\left[\lambda(x) v(x)-\lambda\left(x^{\prime}\right) v\left(x^{\prime}\right)\right]}{\varepsilon^{2}} d \mu\left(x^{\prime}\right) d \mu(x),
\end{aligned}
$$


where $\kappa(\varepsilon)$ is a positive function in $\varepsilon$ that tends to 0 as $\varepsilon \rightarrow 0$. In the above inequality, replace $\lambda$ by $t \lambda$ with $t=\kappa(\varepsilon)^{1 / 2}$, divide by $t$ and then let $\varepsilon \rightarrow 0$, we obtain

$$
\begin{aligned}
& \limsup _{\varepsilon \rightarrow 0} \int \frac{\eta(x)}{c(x, \varepsilon)} \int_{B(x, \varepsilon)} \frac{\lambda(x)\left[d^{2}\left(u(x), u\left(x^{\prime}\right)\right)-\left(v(x)-v\left(x^{\prime}\right)\right)^{2}\right]}{\varepsilon^{2}} d \mu\left(x^{\prime}\right) d \mu(x) \\
& \leq \limsup _{\varepsilon \rightarrow 0}-\int \frac{\eta(x)}{c(x, \varepsilon)} \int_{B(x, \varepsilon)} \frac{\left(v(x)-v\left(x^{\prime}\right)\right)\left[\lambda(x) v(x)-\lambda\left(x^{\prime}\right) v\left(x^{\prime}\right)\right]}{\varepsilon^{2}} d \mu\left(x^{\prime}\right) d \mu(x) \\
& =-\int_{X} \eta(x) d \Gamma_{0}(\lambda v, v)(x),
\end{aligned}
$$

where in the last step we have applied Lemma 4.7. Since $\left|v(x)-v\left(x^{\prime}\right)\right| \leq d\left(u(x), u\left(x^{\prime}\right)\right)$, the first term in the above inequality is non-negative, this proves the claim.

We would like to point out the following Liouville theorem, which follows directly from Lemma 5.1.

Theorem 5.2. Let $X$ be a complete non-compact metric measure space and $Y$ an NPC space. Suppose the approximating energy density $e_{\varepsilon}^{c, Y}$ between $X$ and $Y$ has strong property $\mathcal{C}$. If $u \in K S_{\mathrm{loc}}^{1,2}(X, Y) \cap L^{2}(X, Y)$ is a harmonic mapping, then $u$ is constant.

Proof of Theorem 5.2. Fix a base point $x_{0} \in X$ and define $\rho: X \rightarrow \mathbb{R}$ as

$$
\rho(x)=\max \left\{0, \min \left\{1,2-\frac{1}{R} d\left(x, x_{0}\right)\right\}\right\} .
$$

Then $\rho$ is $\frac{1}{R}$-Lipschitz and $\rho=0$ on $X \backslash B\left(x_{0}, 2 R\right)$ and $\rho=1$ on $B\left(x_{0}, R\right)$. For notational simplicity, we write $B_{r}=B\left(x_{0}, r\right)$. Since $v=d\left(u(\cdot), u\left(x_{0}\right)\right)$ satisfies (5.1),

$$
0 \geq \mathcal{E}_{0}\left(\rho^{2} v, v\right)=\frac{1}{2} \mathcal{E}_{0}\left(\rho^{2}, v^{2}\right)+\int_{X} \rho^{2} d \Gamma_{0}(v, v) .
$$

By the Leibniz rule and Cauchy-Schwartz inequality for Dirichlet forms, we have

$$
\begin{aligned}
\mathcal{E}_{0}\left(\rho^{2}, v^{2}\right)^{2} & =\left(\int_{X} d \Gamma_{0}\left(\rho^{2}, v^{2}\right)\right)^{2}=16\left(\int_{X} \rho v d \Gamma_{0}(\rho, v)\right)^{2} \\
& \leq \int_{X} v^{2} d \Gamma_{0}(\rho, \rho) \cdot \int_{X} \rho^{2} d \Gamma_{0}(v, v) .
\end{aligned}
$$

Consequently, we have

$$
\mathcal{E}_{0}\left(\rho^{2}, v^{2}\right) \geq-4\left(\int_{X} v^{2} d \Gamma_{0}(\rho, \rho)\right)^{1 / 2} \cdot\left(\int_{X} \rho^{2} d \Gamma_{0}(v, v)\right)^{1 / 2} .
$$

Substituting the above estimate in (5.3), we obtain

$$
\begin{gathered}
0 \geq \int_{B_{2 R} \backslash B_{R}} \rho^{2} d \Gamma_{0}(v, v)-2\left(\int_{B_{2 R} \backslash B_{R}} v^{2} d \Gamma_{0}(\rho, \rho)\right)^{\frac{1}{2}} \cdot\left(\int_{B_{2 R} \backslash B_{R}} \rho^{2} d \Gamma_{0}(v, v)\right)^{\frac{1}{2}} \\
+\int_{B_{R}} d \Gamma_{0}(v, v),
\end{gathered}
$$


which is a polynomial, $P(\Psi)=\Psi^{2}-2 b \Psi+c$ with $\Psi=\left(\int_{B_{2} R \backslash B_{R}} \rho^{2} d \Gamma_{0}(v, v)\right)^{\frac{1}{2}}$. Since it has non-positive value, it must hold $b^{2} \geq c$ and so

$$
\int_{B_{R}} d \Gamma_{0}(v, v) \leq \int_{B_{2 R} \backslash B_{R}} v^{2} d \Gamma_{0}(\rho, \rho) \lesssim \frac{1}{R^{2}} \int_{B_{2 R}} v^{2} d \mu
$$

In particular,

$$
\int_{B_{R}} d \Gamma_{0}(v, v) \lesssim \frac{1}{R^{2}} \int_{X} v^{2} d \mu .
$$

Since $v \in L^{2}(X)$, sending $R$ to infinity, we conclude that

$$
\int_{X} d \Gamma_{0}(v, v)=\mathcal{E}_{0}(v, v)=0 .
$$

It follows from the Poincaré inequality that $v$ is constant and hence $u \equiv u\left(x_{0}\right)$ on $X$.

\subsection{Proof of Theorem $\mathbf{1 . 8}$.}

Proposition 5.3. If $u: X \rightarrow Y$ is harmonic, then for each $y_{0} \in Y$, the function $v=d\left(u(\cdot), y_{0}\right): X \rightarrow \mathbb{R}$ is weakly subharmonic, i.e. for each positive Lipschitz function $\lambda: X \rightarrow \mathbb{R}$ with compact support $U \subset \subset X$,

$$
-\mathcal{E}_{0}(\lambda, v) \geq 0
$$

Proof. The passage from (5.1) to subharmonicity is well-known and relies on the fine topology and potential theory; see for instance in $[22,18]$. For the convenience of the readers, we present the proof here.

Let $\psi \in N^{1,2}(X) \cap L^{\infty}(X)$ be nonnegative function with $\operatorname{supp}(\psi) \subset U \subset \subset X$ and let $T_{n}: \mathbb{R} \rightarrow \mathbb{R}$ be the nearest point project of $\mathbb{R}$ onto $\left[\frac{1}{n}, n\right]$. Then the cut-off functions $t_{n}:=T_{n} \circ d\left(\cdot, y_{0}\right)$ and $\frac{1}{t_{n}}$ are $n$-Lipschitz. Thus the truncated functions $v_{n}:=t_{n} \circ u$ and $\frac{1}{v_{n}}$ are in the Sobolev space $N_{l o c}^{1,2}(X)$. Consider the sets

$$
U_{n}:=\left\{x \in U: \frac{1}{n}<v(x)<n\right\}, \quad n \in \mathbb{N} .
$$

For each $n \in \mathbb{N}, U_{n}$ is quasiopen as $v$ is quasicontinuous (see Section 2.4.3). Thus $\left\{U_{n}\right\}_{n \in \mathbb{N}}$ forms a covering of $U$ with quasiopen sets.

By Lemma 2.11, we may find a sequence of nonnegative functions $\left\{\psi_{j}\right\}_{j \in N}$ converging to $\psi$ in $N_{0}^{1,2}(U)$ such that each $\psi_{j}$ is a finite sum of functions in $N_{0}^{1,2}\left(U_{\alpha}\right)$. Without loss of generality, we may assume each $\psi_{j}$ lies in $N_{0}^{1,2}\left(U_{j}\right)$. Then $\lambda_{n}:=\frac{\psi_{n}}{v_{n}} \in N_{0}^{1,2}(U) \cap L^{\infty}(U)$ and $\psi_{n}=\lambda_{n} v$ in $U_{n}$. Moreover, by (5.1), we have

$$
-\mathcal{E}_{0}(\psi, v)=-\lim _{n \rightarrow \infty} \mathcal{E}_{0}\left(\psi_{n}, v\right)=-\lim _{n \rightarrow \infty} \mathcal{E}_{0}\left(\lambda_{n} v, v\right) \geq 0
$$

The proof is complete.

Proof of Theorem 1.8. By Proposition 5.3, the function $v(x)=d\left(u(x), u\left(x_{0}\right)\right), x_{0} \in X$, is weakly subharmonic. By [74, Theorem 1], $v$ is constant and so $u$ is constant as well. 


\section{HARMONIC MAPPING FLOW}

Throughout this section, we assume that $X$ is $\operatorname{RCD}(K, N)$ and $Y$ is NPC. Recall that the distance $D$ on $L^{2}(X, Y)$ is defined as

$$
D^{2}(u, v)=\int_{X} d^{2}(u(x), v(x)) d \mu(x) .
$$

The proof of the following elementary lemma can be found in [46, Corollary 4.1.1].

Lemma 6.1. $L^{2}(X, Y)$ is $N P C$.

Proof of Theorem 1.9. Since $u \mapsto E(u)$ is a lower semicontinuous convex functional on the NPC space $L^{2}(X, Y)$ (by Lemma 6.1), the result follows immediately from [64, Theorem $1.13]$.

Proof of Corollary 1.10. As the Sobolev energy of $u(t)$ decreases, it is uniformly bounded. As $X$ is compact, Theorem 1.9 implies that $u(t)$ has bounded $L^{2}$-norm. By the precompactness of Sobolev spaces, there is subsequence $\left\{u\left(t_{k}\right)\right\}$ that converges in $L^{2}$ as $t_{k} \rightarrow \infty$. By [64, Proposition 2.4], it will converges to an energy minimizer, which is a constant mapping.

Fix an open set $\Omega \subset X$ and we set

$$
\mathcal{K S}_{\phi}^{1,2}(\Omega, Y)=\left\{u \in K S_{\phi}^{1,2}(\Omega, Y): E(u) \leq E(\phi)\right\}
$$

Lemma 6.2. The metric space $\mathcal{K S}_{\phi}^{1,2}(\Omega, Y)$ (equipped with the metric $\left.D\right)$ is $N P C$.

Proof. For each sequence $\left\{u_{k}\right\} \subset \mathcal{K S}_{\phi}^{1,2}(\Omega, Y)$ with $u_{k} \rightarrow u$ in $L^{2}(X, Y)$, we have $E(u) \leq$ $E(\phi)$ by the lower semicontinuity of the Dirichlet energy. Note that

$$
E\left(u_{k}\right)+E(u) \leq 2 E(\phi),
$$

which is uniformly bounded and $u_{k} \rightarrow u$ in $L^{2}(X, Y)$. We may apply Lemma 3.8 to conclude that $u \in \mathcal{K S}_{\phi}^{1,2}(\Omega, Y)$. It remains to show that $\mathcal{K S}_{\phi}^{1,2}(\Omega, Y)$ is a length space, which suffices as $\mathcal{K S}_{\phi}^{1,2}(\Omega, Y)$ then inherits the NPC property from $L^{2}(X, Y)$. However, this is clear since $\mathcal{K S}_{\phi}^{1,2}(\Omega, Y)$ is a convex subset of $L^{2}(X, Y)$ (due to the convexity of the Korevaar-Schoen energy).

It is now possible to apply [64, Theorem 1.13]. Note that the inequality $E(u) \leq E(\phi)$ in the definition of $\mathcal{K S}_{\phi}^{1,2}(\Omega, Y)$ is only a technical requirement for showing $\mathcal{K S}_{\phi}^{1,2}(\Omega, Y)$ is closed. It has no effect on the flow as each time the energy decreases. When $\Omega$ is relatively compact in $X$, by the proof of Theorem 1.1, any minimizing sequence converges to the unique minimizer for the Dirichlet energy of Korevaar and Schoen. Thus we obtain the following theorem via [64, Theorem 1.13].

Proof of Theorem 1.11. This is a direct consequence of Lemma 6.2 and [64, Theorem $1.13]$. 
Acknowledgements. The author would like to thank Prof. Yau Shing-Tung for his insightful comments on the theory of harmonic mappings and for his encouragement on developing the theory in the singular space setting during the 7th ICCM in Beijing 2016, which is the main motivation of this paper. He is grateful to Prof. Jost for kindly sharing his papers $[42,45]$ and for his valuable comments on the background of harmonic mappings, to Prof. Chen for kindly sharing his paper [15], and to Profs. Zhang and Zhu for their interest in this work and for kindly sharing the references [38, 39, 83].

\section{REFERENCES}

[1] S.I. Alber, On n-dimensional problem in the calculus of variations in the large, Sov. Math. Dokl. 5 (1964), 700-704.

[2] S.I. Alber, Spaces of mappings into a manifold with negative curvature, Sov. Math. Dokl. 9 (1967), 6-9.

[3] A.D. Alexandrov, Über eine Verallgemeinerung der Riemannschen Geometrie. (German) Schr. Forschungsinst. Math. 1 (1957), 33-84.

[4] L. Ambrosio, N. Gigli and G. Savaré, Calculus and heat flow in metric measure spaces and applications to spaces with Ricci bounds from below, Invent. Math. 195 (2014), no. 2, 289-391.

[5] L. Ambrosio, N. Gigli and G. Savaré, Metric measure spaces with Riemannian Ricci curvature bounded from below, Duke Math. J. 163 (2014), no. 7, 1405-1490.

[6] L. Ambrosio, N. Gigli and G. Savaré, Bakry-Émery curvature-dimension condition and Riemannian Ricci curvature bounds, Ann. Probab. 43 (2015), no. 1, 339-404.

[7] V.N. Berestovskij and I.G. Nikolaev, Multidimensional generalized Riemannian spaces. Geometry, IV, 165-243, 245-250, Encyclopaedia Math. Sci., 70, Springer, Berlin, 1993.

[8] M. Biroli and U. Mosco, A Saint-Venant type principle for Dirichlet forms on discontinuous media, Ann. Mat. Pura Appl. (4) 169 (1995), 125-181.

[9] A. Björn and J. Björn, Nonlinear potential theory on metric spaces, EMS Tracts in Mathematics, 17. European Mathematical Society (EMS), Zürich, 2011.

[10] A. Björn, J. Björn and V. Latvala, The Cartan, Choquet and Kellogg properties for the fine topology on metric spaces. J. Anal. Math. 135 (2018), no. 1, 59-83.

[11] M.R. Bridson and A. Haefliger, Metric spaces of non-positive curvature. Grundlehren der Mathematischen Wissenschaften [Fundamental Principles of Mathematical Sciences], 319. Springer-Verlag, Berlin, 1999.

[12] L. Capogna and F-H. Lin, Legendrian energy minimizers. I. Heisenberg group target, Calc. Var. Partial Differential Equations 12 (2001), no. 2, 145-171.

[13] S.Y. Cheng, Liouville theorem for harmonic maps, Geometry of the Laplace operator (Proc. Sympos. Pure Math., Univ. Hawaii, Honolulu, Hawaii, 1979), pp. 147-151, Proc. Sympos. Pure Math., XXXVI, Amer. Math. Soc., Providence, R.I., 1980.

[14] J. Cheeger, Differentiability of Lipschitz functions on metric measure spaces, Geom. Funct. Anal. 9 (1999), no. 3, 428-517.

[15] J.Y. Chen, On energy minimizing mappings between and into singular spaces, Duke Math. J. 79 (1995), 77-99. 
[16] G. Daskalopoulos and C. Mese, Harmonic maps from a simplicial complex and geometric rigidity, J. Differential Geom. 78 (2008), no. 2, 269-293.

[17] G. Daskalopoulos and C. Mese, Harmonic maps between singular spaces I, Comm. Anal. Geom. 18 (2010), no. 2, 257-337.

[18] J. Eells and B. Fuglede, Harmonic maps between Riemannian polyhedra. With a preface by M. Gromov, Cambridge Tracts in Mathematics, 142. Cambridge University Press, Cambridge, 2001.

[19] J. Eells and J.H. Sampson, Harmonic mappings of Riemannian manifolds, Amer. J. Math. 86 (1964), 109-160.

[20] M. Erbar, K. Kuwada and K.-T. Sturm, On the equivalence of the entropic curvature-dimension condition and Bochner's inequality on metric measure spaces, Invent. Math. 201 (2015), no. 3, 9931071.

[21] B. Fuglede, Hölder continuity of harmonic maps from Riemannian polyhedra to spaces of upper bounded curvature, Calc. Var. Partial Differential Equations 16 (2003), no. 4, 375-403.

[22] B. Fuglede, Dirichlet problems for harmonic maps from regular domains, Proc. London Math. Soc. (3) 91 (2005), no. 1, 249-272.

[23] B. Fuglede, Harmonic maps from Riemannian polyhedra to geodesic spaces with curvature bounded from above, Calc. Var. Partial Differential Equations 31 (2008), no. 1, 99-136.

[24] M. Fukushima, Y. Oshima and M. Takeda, Dirichlet Forms and Symmetric Markov Processes, in: de Gruyter Studies in Mathematics, vol. 19, Walter de Gruyter \& Co., Berlin, 1994.

[25] M. Giaquinta and E. Giusti, On the regularity of the minima of variational integrals, Acta Math. 148 (1982), 31-46.

[26] M. Giaquinta and E. Giusti, The singular set of the minima of certain quadratic functionals, Ann. Scuola Norm. Sup. Pisa Cl. Sci. (4) 11 (1984), no. 1, 45-55.

[27] N. Gigli and A. Tyulenev, Korevaar-Schoen's energy on strongly rectifiable spaces, preprint 2020.

[28] G. Gregori, Sobolev spaces and harmonic maps between singular spaces, Calc. Var. Part. Diff. Equ. 7 (1998), 1-18.

[29] M. Gromov and R. Schoen, Harmonic maps into singular spaces and p-adic superrigidity for lattices in groups of rank one, Inst. Hautes Études Sci. Publ. Math. No. 76 (1992), 165-246.

[30] C.-Y. Guo and S. Wenger, Area minimizing discs in locally non-compact spaces, Comm. Anal. Geom. 28 (2020), no. 1, 89-112.

[31] R.S. Hamilton, Harmonic maps of manifolds with boundary, Lecture Notes in Mathematics, Vol. 471. Springer-Verlag, Berlin-New York, 1975.

[32] P. Hartman, On homotopic harmonic maps, Canad. J. Math. 19 (1967), 673-687.

[33] J. Heinonen, T. Kilpeläinen and O. Martio, Nonlinear potential theory of degenerate elliptic equations, Unabridged republication of the 1993 original. Dover Publications, Inc., Mineola, NY, 2006.

[34] J. Heinonen and P. Koskela, Quasiconformal maps in metric spaces with controlled geometry, Acta Math. 181 (1998), 1-61.

[35] J. Heinonen, P. Koskela, N. Shanmugalingam and J.T. Tyson, Sobolev spaces on metric measure spaces: an approach based on upper gradients, New Mathematical Monographs, 27, Cambridge University Press, Cambridge, 2015.

[36] S. Hildebrandt, J. Jost and K.O. Widman, Harmonic mappings and minimal submanifolds, Invent. Math. 62 (1980/81), no. 2, 269-298. 
[37] S. Hildebrandt, H. Kaul and K.O. Widman, An existence theorem for harmonic mappings of Riemannian manifolds, Acta Math. 138 (1977), no. 1-2, 1-16.

[38] J.-C. Huang, Some problems of nonlinear analysis on Alexandrov spaces, PhD thesis, Sun Yat-sen University, 2016.

[39] J.-C. Huang and H.-C. Zhang, Harmonic maps between Alexandrov spaces, J. Geom. Anal. 27 (2017), no. 2, 1355-1392.

[40] W. Ishizuka and C.Y. Wang, Harmonic maps from manifolds of $L^{\infty}$-Riemannian metrics, Calc. Var. Partial Differential Equat. 32:3 (2008), 387-405.

[41] R. Jiang, Cheeger-harmonic functions in metric measure spaces revisited, J. Funct. Anal. 266 (2014), no. 3, 1373-1394.

[42] J. Jost, Equilibrium maps between metric spaces, Calc. Var. Partial Differential Equations 2 (1994), no. 2, 173-204.

[43] J. Jost, Convex functionals and generalized harmonic maps into spaces of nonpositive curvature, Comment. Math. Helv. 70 (1995), no. 4, 659-673.

[44] J. Jost, Generalized harmonic maps between metric spaces, Geometric analysis and the calculus of variations, 143-174, Int. Press, Cambridge, MA, 1996.

[45] J. Jost, Generalized Dirichlet forms and harmonic maps, Calc. Var. Partial Differential Equations 5 (1997), no. 1, 1-19.

[46] J. Jost, Nonpositive curvature: geometric and analytic aspects, Lectures in Mathematics ETH Zürich. Birkhäuser Verlag, Basel, 1997.

[47] J. Jost and M. Meier, Boundary regularity for minima of certain quadratic functionals, Math. Ann. 262 (1983), no. 4, 549-561.

[48] J. Jost, Nonlinear Dirichlet forms, New directions in Dirichlet forms, J. Jost and W. Kendll and U. Mosco and M. Röckner and K.-T. Sturm (editor), 1-47, International Press/AMS, 1998.

[49] T. Kilpeläinen and J. Malý, Supersolutions to degenerate elliptic equation on quasi open sets, Comm. Partial Differential Equations 17 (1992), no. 3-4, 371-405.

[50] J. Kinnunen and N. Shanmugalingam, Regularity of quasi-minimizers on metric spaces, Manuscripta Math. 105 (2001), no. 3, 401-423.

[51] N.J. Korevaar and R. Schoen, Sobolev spaces and harmonic maps for metric space targets, Comm. Anal. Geom. 1 (1993), no. 3-4, 561-659.

[52] N.J. Korevaar and R. Schoen, Global existence theorems for harmonic maps to non-locally compact spaces, Comm. Anal. Geom. 5 (1997), no. 2, 333-387.

[53] P. Koskela and P. MacManus, Quasiconformal mappings and Sobolev spaces, Studia Math. 131 (1998), no. 1, 1-17.

[54] P. Koskela, K. Rajala and N. Shanmugalingam, Lipschitz continuity of Cheeger-harmonic functions in metric measure spaces, J. Funct. Anal. 202 (2003), no. 1, 147-173.

[55] P. Koskela, N. Shanmugalingam and J.T. Tyson, Dirichlet forms, Poincaré inequalities, and the Sobolev spaces of Korevaar and Schoen, Potential Anal. 21 (2004), no. 3, 241-262.

[56] P. Koskela and Y. Zhou, Geometry and analysis of Dirichlet forms, Adv. Math. 231 (2012), no. 5, 2755-2801.

[57] K. Kuwae and T. Shioya, Sobolev and Dirichlet spaces over maps between metric spaces, J. Reine Angew. Math. 555 (2003), 39-75. 
[58] K. Kuwae and K.-T. Sturm, On a Liouville type theorem for harmonic maps to convex spaces via Markov chains, Proceedings of RIMS Workshop on Stochastic Analysis and Applications, 177-191, RIMS Kokyuroku Bessatsu, B6, Res. Inst. Math. Sci. (RIMS), Kyoto, 2008.

[59] F.-H. Lin, Analysis on singular spaces, Collection of papers on geometry, analysis and mathematical physics, 114-126, World Sci. Publ., River Edge, NJ, 1997.

[60] J. Lott and C. Villani, Ricci curvature for metric-measure spaces via optimal transport, Ann. of Math. (2) 169 (2009), no. 3, 903-991.

[61] A. Lytchak and S. Wenger, Area minimizing discs in metric spaces, Arch. Rational. Mech. Anal. 223 (2017), no. 3, 1123-1182.

[62] A. Lytchak and S. Wenger, Regularity of harmonic discs in spaces with quadratic isoperimetric inequality, Calc. Var. Partial Differential Equations 55 (2016), no. 4, Paper No. 98, 19 pp.

[63] J. Malý and W.P. Ziemer, Fine regularity of solutions of elliptic partial differential equations. Mathematical Surveys and Monographs, 51. American Mathematical Society, Providence, RI, 1997.

[64] Uwe F. Mayer, Gradient flows on nonpositively curved metric spaces and harmonic maps, Comm. Anal. Geom. 6 (1998), 199-253.

[65] C.B. Morrey, The problem of plateau on a Riemannian manifold, Ann. Math. (2) 49 (1948), 807-851.

[66] T. Rajala, Local Poincaré inequalities from stable curvature conditions on metric spaces, Calc. Var. Partial Differential Equations 44 (2012), no. 3-4, 477-494.

[67] R. Schoen and K. Uhlenbeck, A regularity theory for harmonic maps, J. Differential Geom. 17 (1982), no. $2,307-335$.

[68] R. Schoen and K. Uhlenbeck, Boundary regularity and the Dirichlet problem for harmonic maps, J. Differential Geom. 18 (1983), no. 2, 253-268.

[69] R. Schoen and S.-T. Yau, Harmonic maps and the topology of stable hypersurfaces and manifolds with non-negative Ricci curvature, Comment. Math. Helv. 51 (1976), no. 3, 333-341.

[70] R. Schoen and S.-T. Yau, Lectures on harmonic maps, Conference Proceedings and Lecture Notes in Geometry and Topology, II. International Press, Cambridge, MA, 1997.

[71] N. Shanmugalingam, Newtonian spaces: an extension of Sobolev spaces to metric measure spaces, Rev. Mat. Iberoamericana 16 (2000), no. 2, 243-279.

[72] N. Shanmugalingam, Harmonic functions on metric spaces, Illinois J. Math. 45 (2001), no. 3, 10211050.

[73] Y.-G. Shi, A partial regularity result of harmonic maps from manifolds with bounded measurable Riemannian metrics, Comm. Anal. Geom. 4 (1996), no. 1-2, 121-128.

[74] K.-T. Sturm, Analysis on local Dirichlet spaces. I. Recurrence, conservativeness and $L^{p}$-Liouville properties, J. Reine Angew. Math. 456 (1994), 173-196.

[75] K.-T. Sturm, Diffusion processes and heat kernels on metric spaces, Ann. Probab. 26 (1998), no. 1, $1-55$.

[76] K.-T. Sturm, Nonlinear Markov operators associated with symmetric Markov kernels and energy minimizing maps between singular spaces, Calc. Var. 12 (2001), 317-357.

[77] K.-T. Sturm, Nonlinear martingale theory for processes with values in metric spaces of nonpositive curvature, Ann. Probab. 30 (2002), 1195-1222.

[78] K.-T. Sturm, A semigroup approach to harmonic maps, Potential Anal. 23 (2005), no. 3, 225-277. 
[79] K.-T. Sturm, On the geometry of metric measure spaces. I, II, Acta Math. 196 (2006), no.1.

[80] S.-T. Yau, Some function-theoretic properties of complete Riemannian manifold and their applications to geometry, Indiana Univ. Math. J. 25 (1976), no. 7, 659-670.

[81] H.-C. Zhang, X. Zhong and X.-P. Zhu, Quantitative gradient estimates for harmonic maps into singular spaces, Sci. China Math. 62 (2019), no. 11, 2371-2400.

[82] H.-C. Zhang and X.-P. Zhu, Lipschitz continuity of harmonic maps between Alexandrov spaces, Invent. Math. 211 (2018), no. 3, 863-934.

[83] H.-C. Zhang and X.-P. Zhu, Regularity of harmonic maps on Alexandrov spaces, preprint 2016.

(Chang-Yu Guo) Research Center for Mathematics and Interdisciplinary Sciences, Shandong University 266237, Qingdao, P. R. China

Email address: changyu.guo@sdu.edu.cn 